\title{
Efetividade da terapia por oxigenação de membrana extracorpórea (ECMO) em pacientes críticos com COVID-19
}

\author{
Effectiveness of extracorporeal membrane oxygenation (ECMO) therapy in critically patients with \\ COVID-19
}

Eficacia de la terapia de oxigenación por membrana extracorpórea (ECMO) en pacientes críticos con COVID-19

\author{
Stephanie Zambrano Lavezzo \\ ORCID: https://orcid.org/0000-0003-3748-0039 \\ Universidade São Judas Tadeu, Brasil \\ E-mail: stephanielavezzo@hotmail.com \\ Samantha Ellen Beltrame \\ ORCID: https://orcid.org/0000-0001-6703-7826 \\ Universidade São Judas Tadeu, Brasil \\ E-mail: samanthaellensb@hotmail.com \\ Carolina Heitmann Mares Azevedo Ribeiro \\ ORCID: https://orcid.org/0000-0002-9457-2733 \\ Universidade Federal do Pará, Brasil \\ E-mail: chribeiro@ufpa.br \\ Ana Cristina Lo Prete \\ ORCID: https://orcid.org/0000-0002-2874-5296 \\ Universidade São Judas Tadeu, Brasil \\ E-mail: analoprete@gmail.com
}

\begin{abstract}
Resumo
Casos graves de COVID-19 podem necessitar de terapias de suporte de oxigênio, como a utilização de Membrana de Oxigenação Extracorpórea (ECMO) e ventilação mecânica (VM). A ECMO é uma terapia usada para suporte temporário em casos de falência da função pulmonar e/ou cardíaca. Esta revisão integrativa objetivou avaliar a literatura científica sobre a efetividade da ECMO em pacientes críticos com COVID-19. Foram incluídos na presente revisão artigos coletados do PubMed, publicados no período de dezembro de 2019 a junho de 2021, utilizando-se os descritores "COVID-19" e "ECMO". Foram excluídos artigos que não apresentavam resultados sobre a avaliação da relação da ECMO com a COVID-19 e os que se enquadravam na categoria de modelo de carta ao editor ou revisões narrativas. Para a discussão de forma integrada, os artigos foram agrupados em uma tabela contendo título, objetivo, método, metodologia, resultados e conclusão. Foram incluídos para a análise integrativa, 36 artigos. Foi possível evidenciar uma maior eficácia da ECMO nos pacientes mais jovens ( $<71$ anos), com maior gravidade da doença e nos que apresentavam comorbidades. Porém, a terapia apresenta certos tipos de complicações, como alto risco de trombose e falhas no circuito, o que podem levar a um desmame malsucedido da ECMO. A terapia com ECMO pode auxiliar pacientes graves com COVID-19 em determinadas condições, embora possa aumentar o risco de trombose, sendo necessários mais estudos para um melhor esclarecimento.
\end{abstract}

Palavras-chave: COVID-19; Oxigenação por membrana extracorpórea; Eficácia.

\begin{abstract}
Severe cases of COVID-19 may require oxygen support therapies, such as the use of Extracorporeal Oxygenation Membrane (ECMO) and mechanical ventilation (MV). ECMO is a therapy used for temporary support in cases of pulmonary and/or cardiac function failure. This integrative review aimed to assess the scientific literature on the effectiveness of ECMO in critically patients with COVID-19. Articles collected from PubMed, published from December 2019 to June 2021, using the descriptors "COVID-19" and "ECMO" were included in this review. Articles that did not present results on the assessment of the relation between ECMO and COVID-19 and those that fell into the category of letter to the editor model or narrative reviews were excluded. For an integrated discussion, the articles were grouped in a table containing title, objective, method, methodology, results and conclusion. 36 articles were included for the integrative analysis. It was possible to evidence a greater efficacy of ECMO in younger patients $(<71$ years), with greater disease severity and in those with comorbidities. However, the therapy has certain types of complications, such as a high risk of thrombosis and circuit failure, which can lead to an unsuccessful weaning from ECMO. ECMO therapy can help critically patients with COVID-19 in certain conditions, although it may increase the risk of thrombosis, and further studies are needed for better clarification.
\end{abstract}


Keywords: COVID-19; Extracorporeal membrane oxygenation; Efficiency.

\section{Resumen}

Los casos graves de COVID-19 pueden requerir terapias de apoyo de oxígeno, como el uso de oxigenación por membrana extracorpórea (ECMO) y ventilación mecánica (VM). ECMO es una terapia utilizada como soporte temporal en casos de falla de la función pulmonar y/o cardíaca. Esta revisión integradora tuvo como objetivo evaluar la literatura científica sobre la efectividad de ECMO en pacientes críticos con COVID-19. En esta revisión se incluyeron artículos recopilados de PubMed, publicados entre diciembre de 2019 y junio de 2021, utilizando los descriptores "COVID-19" y "ECMO". Se excluyeron los artículos que no presentaron resultados sobre la evaluación de la relación de ECMO con el COVID-19 y aquellos que encajan en la categoría de modelo de carta al editor o reseñas narrativas. Para una discusión integrada, los artículos fueron agrupados en una tabla que contenía título, objetivo, método, metodología, resultados y conclusión. Para el análisis integrador se incluyeron 36 artículos. Se pudo evidenciar una mayor efectividad de ECMO en pacientes más jóvenes (< 71 años), con mayor gravedad de la enfermedad y en aquellos con comorbilidades. Sin embargo, la terapia tiene ciertos tipos de complicaciones, como un alto riesgo de trombosis y fallas en el circuito, que pueden conducir a un destete ECMO fallido. La terapia ECMO puede ayudar a los pacientes críticos con COVID-19 en ciertas condiciones, aunque puede aumentar el riesgo de trombosis, y se necesitan más estudios para obtener más aclaraciones.

Palabras clave: COVID-19; Oxigenación por membrana extracorpórea; Eficacia.

\section{Introdução}

Em dezembro de 2019, a Organização Mundial da Saúde (OMS) foi informada do surto de casos de uma pneumonia de etiologia não identificada em Wuhan, na China. No final de janeiro de 2020 foi confirmado que se tratava da Síndrome Respiratória Aguda Grave Coronavírus 2 (SARS-COV-2), pertencente à família dos coronavírus, nomeada como a COVID-19, que rapidamente se expandiu pelo mundo (Wang et al., 2020). Até setembro de 2021, dados atualizados disponibilizados pela Universidade de Medicina John Hopkins situada nos Estados Unidos indicaram que 225.725 .790 casos foram registrados mundialmente, incluindo 4.647.639 de mortes causadas pelo vírus (Universidade John Hopkins, 2021).

A COVID-19 afeta diretamente o trato respiratório do indivíduo, causando uma série de infecções no mesmo (Umakanthan et al., 2020). Segundo o Jornal de Cardiologia Brasileiro (2021), de 15 a 20\% dos pacientes diagnosticados com a SARS-COV-2 podem evoluir com a forma mais grave da doença, progredindo muitas vezes para a Síndrome da Angústia Respiratória Aguda (SARA), definida como hipoxemia secundária à um início rápido de edema pulmonar não cardiogênico, abrangendo lesões pulmonares, incluindo pneumonia, sepse, choque não cardiogênico e traumas (Ribeiro, 2020; Williams, 2021).

Nestes casos de agravamento, ocorre a internação do paciente na Unidade de Terapia Intensiva (UTI), sendo este tratado com o uso de medicamentos, Membrana de Oxigenação Extracorpórea (ECMO) e ventilação mecânica (MV), quando necessários (Kowalewski et al., 2020).

A ECMO é uma modalidade de suporte de vida extracorpóreo que possibilita suporte temporário à falência da função pulmonar e/ou cardíaca, refratária ao tratamento convencional. Indicada em casos de insuficiência respiratória hipoxêmica ou hipercapnica e choque cardiogênico ou não cardiogênico. Seu uso de forma venovenosa (VV-ECMO) é escolhido em casos de insuficiência respiratória com função cardíaca preservada, enquanto a ECMO venoarterial é indicada para fornecer suporte cardíaco em casos com função pulmonar preservada ou não (Chaves et al., 2019).

O circuito ECMO consiste em uma bomba de sangue, oxigenador, no qual ocorrem as trocas gasosas entre oxigênio e gás carbônico, drenagem, cânulas de retorno, fluxo, sensores de pressão, trocador de calor (para o resfriamento ou aquecimento) e pontos de acesso arterial e venoso para a coleta de sangue no circuito (Chaves et al., 2019).

O uso desta terapia tornou-se mais conhecido depois da onda de casos de COVID-19 começar, porém esta técnica é utilizada há anos e foi criada em 1953, pelo médico cirurgião estadunidense John Gibbon e aperfeiçoada ao longo dos anos com apoio do crescimento da tecnologia (Hill,1982). 
Muitos artigos vêm sendo publicados analisando a relação da COVID-19 com o uso da ECMO. Deste modo, o presente trabalho tem como objetivo revisar de forma integrativa os dados da literatura científica que avaliam a efetividade da terapia de oxigenação por membrana extracorpórea (ECMO) em pacientes críticos com COVID-19.

\section{Metodologia}

Trata-se de uma revisão integrativa da literatura. A análise foi realizada por meio do cruzamento das palavras-chave disponíveis nos Descritores em Ciências da Saúde (DECS). Foram usados os descritores "COVID-19" e "Extracorporeal Oxygenation Membrane", nas línguas portuguesa e inglesa.

O trabalho tem como alvo a seguinte questão: a terapia de oxigenação por membrana extracorpórea (ECMO) é efetiva em pacientes críticos com COVID-19?

As buscas foram realizadas na base de dados Medical Literature Analysis and Retrieval System on-line (PUBMED), uma vez que esta base permite buscas simultâneas nas principais fontes nacionais e internacionais.

Para realizar essa revisão integrativa, considerou-se a análise de Koche (2011) sobre fundamentos de metodologia científica e Silva et al (2022) sobre revisão integrativa na COVID-19.

A pesquisa englobou artigos publicados no período de dezembro de 2019 a junho de 2021, que apresentaram o texto na íntegra de forma gratuita. Foram adicionados filtros para a seleção do tipo de publicação: estudo clínico, ensaio clínico, estudo observacional, estudo multicêntrico, meta-análise, revisão sistemática e diretriz de prática. A seleção resultou em 87 artigos.

Como critérios de exclusão, foram retirados os estudos que não apresentavam resultados sobre avaliação da relação da ECMO com a COVID-19 e os que se enquadravam na categoria de modelo de carta ao editor, por não se tratar de modelo de forte evidência científica, ou revisões narrativas, por se tratar de artigos que compilam dados de outros artigos, evitando duplicação de informações.

Após aplicação desses critérios o trabalho resultou na análise em pares de 36 artigos, os quais foram utilizados para esta revisão de forma integrada (Figura 1).

Figura 1. Fluxograma da seleção dos trabalhos.

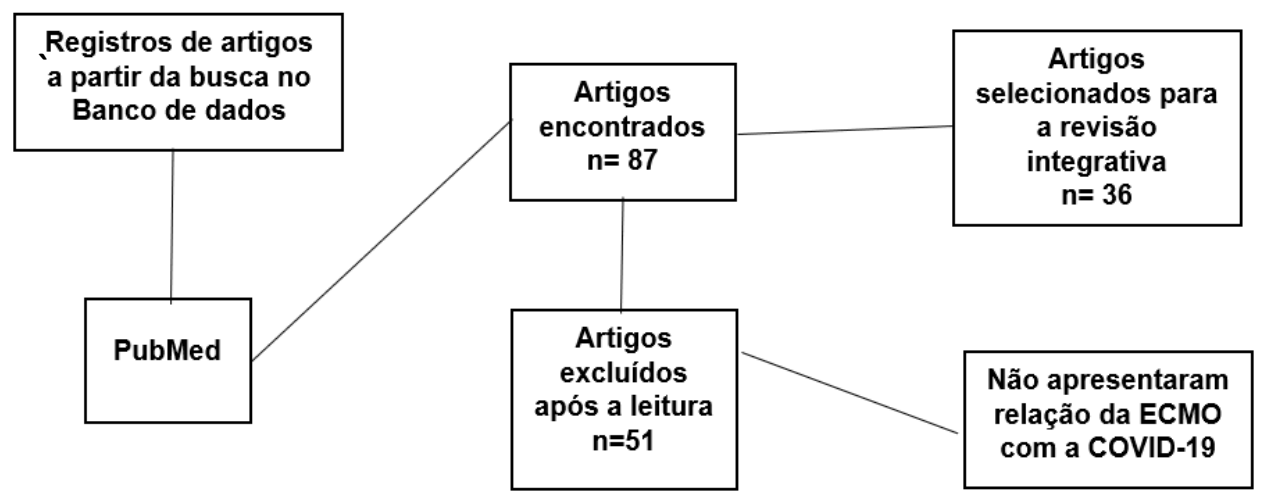

Fonte: Autores (2022).

\section{Resultados}

Seguindo os critérios de seleção estabelecidos, foram incluídos no presente estudo 36 artigos relacionados à ECMO em pacientes críticos com COVID-19. 
Os resultados estão apresentados no Quadro 1, no qual se encontram título, objetivo, método, metodologia, resultado e conclusão dos artigos selecionados.

Quadro 1 - Perfil e características dos artigos selecionados.

\begin{tabular}{|c|c|c|c|c|c|c|}
\hline & ARTIGO & OBJETIVO & $\begin{array}{c}\text { DESENHO DO } \\
\text { ESTUDO }\end{array}$ & METODOLOGIA & RESULTADO & CONCLUSÃO \\
\hline 01 & $\begin{array}{l}\text { Cytokine adsorption in } \\
\text { patients with severe } \\
\text { COVID-19 pneumonia } \\
\text { requiring } \\
\text { extracorporeal membrane } \\
\text { oxygenation. } \\
\text { (RIEDER } \text { et al., 2020). }\end{array}$ & $\begin{array}{l}\text { Avaliar se a adsorção de } \\
\text { citocinas é benéfica em } \\
\text { pacientes críticos com a } \\
\text { COVID-19 que } \\
\text { necessitam de suporte de } \\
\text { oxigenação de } \\
\text { membrana extracorpórea } \\
\text { veno-venosa (vv- } \\
\text { ECMO). }\end{array}$ & $\begin{array}{l}\text { Multicêntrico } \\
\text { randomizado } \\
\text { clínico controlado }\end{array}$ & $\begin{array}{l}\text { Randomizaram pacientes com } \\
\text { COVID-19 que necessitavam de } \\
\text { VV-ECMO. Durante o uso, estes } \\
\text { pacientes receberam adsorção de } \\
\text { citocinas e foram acompanhados } \\
\text { por } 72 \mathrm{~h} \text {. }\end{array}$ & $\begin{array}{l}\text { Ocorreu redução da IL- } 6 \text { em } 75 \% \text { dos } \\
\text { pacientes após } 72 \text { horas em comparação } \\
\text { com a medição da linha de base e o } \\
\text { tempo para a explantação de ECMO bem } \\
\text { sucedida dentro de } 30 \text { dias. }\end{array}$ & $\begin{array}{l}\text { Houve expressiva redução da } \\
\text { inflamação após } 72 \mathrm{~h} \text { de uso de } \\
\text { ECMO com adsorção de } \\
\text { citocinas. }\end{array}$ \\
\hline 02 & $\begin{array}{l}\text { Extracorporeal membrane } \\
\text { oxygenation (ECMO) in } \\
\text { patients with COVID-19: a } \\
\text { rapid systematic review of } \\
\text { case studies } \\
\text { (HU et al., 2020). }\end{array}$ & $\begin{array}{l}\text { Esclarecer o uso da } \\
\text { ECMO em pacientes } \\
\text { com a COVID- } 19 .\end{array}$ & $\begin{array}{c}\text { Revisão } \\
\text { sistemática }\end{array}$ & $\begin{array}{l}\text { Três revisores avaliaram relatos de } \\
\text { casos de pacientes com COVID-19 } \\
\text { grave, tratados com ECMO. }\end{array}$ & $\begin{array}{l}\text { De } 72 \text { pacientes, } 52,8 \% \text { morreram, } \\
18,0 \% \text { ainda estavam em tratamento } \\
\text { ECMO, } 16,7 \% \text { estavam em recuperação, } \\
9,7 \% \text { foram recuperados e } 2,8 \% \\
\text { permaneceram hospitalizados. }\end{array}$ & $\begin{array}{l}\text { A ECMO estabiliza e aumenta } \\
\text { a sobrevivência de pacientes } \\
\text { graves com COVID-19, mas a } \\
\text { utilidade para redução da } \\
\text { mortalidade SARA foi limitada. }\end{array}$ \\
\hline 03 & $\begin{array}{l}\text { Thrombotic circuit } \\
\text { complications during } \\
\text { venovenous extracorporeal } \\
\text { membrane oxygenation in } \\
\text { COVID-19. } \\
\text { (BEMTGEN et al., 2020). }\end{array}$ & 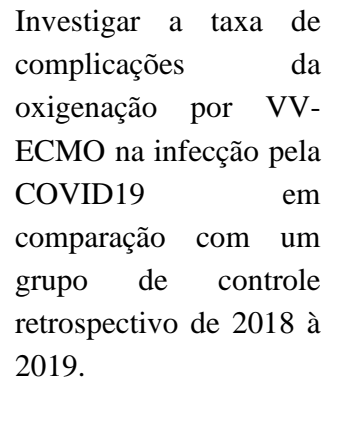 & $\begin{array}{l}\text { Unicêntrico } \\
\text { prospectivo }\end{array}$ & $\begin{array}{l}\text { Dados de todos os pacientes em V- } \\
\mathrm{V} \text { ECMO tratados em uma UTI, } \\
\text { onde todos os circuitos da ECMO } \\
\text { foram verificados uma vez por dia } \\
\text { por um perfusionista, três vezes por } \\
\text { dia por cada enfermeiro e um } \\
\text { médico para formações visíveis de } \\
\text { trombos, depósitos lipídicos e } \\
\text { alterações no rolamento da cabeça } \\
\text { da bomba. }\end{array}$ & $\begin{array}{l}\text { Foram analisados } 66 \text { pacientes V-V } \\
\text { ECMO, onze pacientes que foram } \\
\text { testados positivos para SARS- } \\
\text { CoV-2 e } 55 \text { pacientes atendidos no } \\
\text { hospital. A sobrevida de } 28 \text { dias foi de } \\
72,7 \% \text { no grupo COVID- } 19 \text { e } 58,2 \% \text { para } \\
\text { o grupo não COVID-19. Foi obtido como } \\
\text { resultado uma taxa significativamente } \\
\text { maior de complicações trombóticas no } \\
\text { circuito ECMO de pacientes COVID-19, }\end{array}$ & $\begin{array}{l}\text { A formação de trombos no } \\
\text { circuito extracorpóreo em } \\
\text { pacientes COVID-19 é comum } \\
\text { e potencialmente fatal. Em } \\
\text { comparação com o V-V ECMO } \\
\text { usual, o risco é maior e, por } \\
\text { isso, é necessário um } \\
\text { acompanhamento especial para } \\
\text { evitar complicações. }\end{array}$ \\
\hline
\end{tabular}




\begin{tabular}{|c|c|c|c|c|c|c|}
\hline & & & & $\begin{array}{l}\text { Foram coletados } \\
\text { demográficos (idade, sexo) e } \\
\text { características da linha de base } \\
\text { relacionadas ao V-V ECMO } \\
\text { (duração, indicação, tamanho da } \\
\text { cânula), estratégia anticoagulação, } \\
\text { medições de dímeros-D, sobrevida } \\
\text { à descarga e presença de } \\
\text { complicações relacionadas ao } \\
\text { dispositivo V-V ECMO. }\end{array}$ & $\begin{array}{l}\text { especialmente trombose na cabeça da } \\
\text { bomba, em comparação com pacientes } \\
\text { não COVID-19 (63,6\% vs. } 18,2 \%) \text {. }\end{array}$ & \\
\hline 04 & $\begin{array}{l}\text { COVID-19 and } \\
\text { pneumothorax: } \\
\text { multicentre retrospective } \\
\text { case series } \\
\text { (MARTINELLI et al., } \\
\text { 2020). }\end{array}$ & $\begin{array}{lr}\text { Descrever } & \text { as } \\
\text { características clínicas } \\
\text { dos pacientes com } \\
\text { pneumotórax } & \mathrm{e} \\
\text { pneumodiastino, } & \mathrm{e} \\
\text { considerar se } & \mathrm{o} \\
\text { desenvolvimento } & \mathrm{de} \\
\text { pneumotórax pode } & \mathrm{ser} \\
\text { usado como um } & \mathrm{de} \\
\text { marcador } & \mathrm{de} \\
\text { prognóstico ruim. } & \end{array}$ & $\begin{array}{l}\text { Multicêntrico } \\
\text { retrospectivo }\end{array}$ & $\begin{array}{l}\text { Os critérios de inclusão limitaram- } \\
\text { se ao diagnóstico da COVID-19 e à } \\
\text { presença de pneumotórax ou } \\
\text { pneumomediastino, permitindo } 28 \\
\text { dias de acompanhamento pós- } \\
\text { pneumotórax em cada caso. } \\
\text { Os detalhes obtidos no registro } \\
\text { medical incluíram demografia, } \\
\text { histórico médico passado, } \\
\text { investigações laboratoriais, manejo } \\
\text { clínico, progresso do paciente e } \\
\text { sobrevivência. }\end{array}$ & $\begin{array}{l}\text { No estudo foram incluídos } 71 \text { pacientes, } \\
\text { dos quais } 60 \text { tinham pneumotórax e } 11 \\
\text { tiveram pneumomediastino sozinho. } \\
\text { Alguns pacientes desenvolveram sua } \\
\text { complicação enquanto entubados e } \\
\text { ventilados, com ou sem ECMO. Dos } 71 \\
\text { pacientes, } 12 \text { usaram a ECMO, sendo que } \\
8 \text { sobreviveram. }\end{array}$ & $\begin{array}{l}\text { Não houve diferença } \\
\text { significativa na aposta de } \\
\text { sobrevivência } \\
\text { pneumotórax, apenas com } \\
\text { ventilação invasiva em } \\
\text { comparação com aqueles que } \\
\text { têm suporte ao ECMO, sendo as } \\
\text { duas terapias eficazes } \\
\text { Pacientes com } \geq 70 \text { anos } \\
\text { tiveram uma sobrevida } \\
\text { significativamente menor de } 28 \\
\text { dias do que os mais jovens. }\end{array}$ \\
\hline 05 & $\begin{array}{l}\text { The role of extracorporeal } \\
\text { life support for patients } \\
\text { with COVID-19: } \\
\text { Preliminary results from a } \\
\text { statewide experience. } \\
\text { (SULTAM et al., 2021). }\end{array}$ & $\begin{array}{l}\text { Há uma escassez de } \\
\text { dados clínicos sobre } \\
\text { pacientes gravemente } \\
\text { doentes com COVID-19 } \\
\text { que requerem ECMO. }\end{array}$ & $\begin{array}{c}\text { Revisão } \\
\text { sistemática }\end{array}$ & $\begin{array}{l}\text { Foram obtidos dados clínicos dos } \\
10 \text { primeiros pacientes em todo } \\
\text { estado, gravemente doentes com } \\
\text { COVID-19 que necessitaram de } \\
\text { ECMO. Seus achados laboratoriais } \\
\text { e clínicos, incluindo seu curso } \\
\text { clínico, tempo para ECMO e } \\
\text { recuperação foram obtidos. }\end{array}$ & $\begin{array}{l}\text { Idade variou de } 31 \text { à } 62 \text { anos, sete com } \\
\text { comorbidades. Todos foram canulados } \\
\text { na configuração venosa. Oito }(80 \%) \\
\text { foram liberados entre o } 7 \text { e } 11 \text { dias, } 1 \\
\text { ( } 10 \%) \text { está atualmente em um curso para } \\
\text { "desmamar" e } 1 \text { ( } 10 \%) \text { morreu após } 9 \text { dias } \\
\text { em ECMO de disfunção multiplos órgãos. }\end{array}$ & $\begin{array}{l}\text { Por ter ocorrido apenas } 1 \text { óbito } \\
\text { a terapia foi considerada eficaz } \\
\text { nesta fase inicial, porém haja } \\
\text { riscos de complicações como } \\
\text { tromboembolismo, hemorragias } \\
\text { e infecções. }\end{array}$ \\
\hline 06 & $\begin{array}{l}\text { Multi-system inflammatory } \\
\text { syndrome in children \& }\end{array}$ & $\begin{array}{llr}\text { Avaliar os casos } \\
\text { notificados } & \text { em } & \text { crianças }\end{array}$ & $\begin{array}{l}\text { Revisão } \\
\text { sistemática }\end{array}$ & $\begin{array}{l}\text { Foi realizada busca sistemática no } \\
\text { banco de dados eletrônico }\end{array}$ & $\begin{array}{l}\text { Pacientes com MIS-C apresentaram } \\
\text { gravidade da doença, com } 68 \% \text { dos casos }\end{array}$ & $\begin{array}{l}\text { O MIS-C parece ser uma } \\
\text { condição de alta gravidade, com }\end{array}$ \\
\hline
\end{tabular}




\begin{tabular}{|c|c|c|c|c|c|c|}
\hline & $\begin{array}{l}\text { adolescents (MIS-C): A } \\
\text { systematic review of } \\
\text { clinical features and } \\
\text { presentation. } \\
\\
\text { (RADIA et al., 2020). }\end{array}$ & $\begin{array}{l}\text { e adolescentes da } \\
\text { síndrome inflamatória } \\
\text { multissistêmica (MIS- } \\
\text { C) com associação } \\
\text { temporal com a } \\
\text { COVID-19. }\end{array}$ & & $\begin{array}{l}\text { MEDLINE de 1/12/2019 a } \\
31 / 05 / 2020 \text { sobre a COVID-19 e } \\
\text { crianças, adolescentes, recém- } \\
\text { nascidos ou bebês. Foi empregado } \\
\text { um formulário estruturado de } \\
\text { extração de dados para padronizar a } \\
\text { identificação e recuperação de } \\
\text { dados de manuscritos. }\end{array}$ & $\begin{array}{l}\text { necessitando de UTI; } 63 \% \text { necessitaram } \\
\text { de apoio inotrópico; } 28 \% \text { casos que } \\
\text { necessitam de algum tipo de suporte } \\
\text { respiratório, e } 31 \text { necessitaram de ECMO. } \\
\text { Ocorreram } 12(1,5 \%) \text { mortes, as quais } 7 \\
\text { estavam em ECMO, } 2 \text { morreram após um } \\
\text { AVC e } 3 \text { não tiveram causa da morte } \\
\text { declarada. }\end{array}$ & $\begin{array}{l}68 \% \text { dos casos tendo exigido } \\
\text { apoio de cuidados críticos, } \\
\text { incluindo a ECMO. Apesar das } \\
\text { mortes de } 1.5 \% \text {, a causa para } \\
\text { cada caso não foi conhecida, } \\
\text { com } 7 \text { crianças em suporte } \\
\text { ECMO. Desse modo, a terapia } \\
\text { foi considerada eficaz. }\end{array}$ \\
\hline 07 & $\begin{array}{l}\text { COVID-19 PICU } \\
\text { guidelines: for high- and } \\
\text { limited-resource settings. } \\
\text { (KACHE } \text { et al., 2020). }\end{array}$ & $\begin{array}{l}\text { Apresentar estratégias } \\
\text { de gestão para pacientes } \\
\text { pediátricos gravemente } \\
\text { doentes com COVID-19 } \\
\text { em configurações de } \\
\text { recursos altos e } \\
\text { limitados. }\end{array}$ & $\begin{array}{c}\text { Revisão } \\
\text { Sistemática }\end{array}$ & $\begin{array}{l}\text { Rever evidências disponíveis sobre } \\
\text { evidências para o cuidado de } \\
\text { crianças gravemente doentes com } \\
\text { infecção por SARS-CoV-2. Quando } \\
\text { faltaraam as evidências, essas } \\
\text { lacunas foram substituídas por } \\
\text { diretrizes baseadas em consenso. }\end{array}$ & $\begin{array}{l}\text { Esse processo gerou } 44 \text { recomendações } \\
\text { relacionadas aos pacientes pediátricos } \\
\text { com COVID-19 que apresentam } \\
\text { problemas respiratórios ou insuficiência, } \\
\text { sepse ou choque séptico, parada } \\
\text { cardiopulmonar, MIS-C e aqueles que } \\
\text { necessitam de terapias adjuvantes, ou } \\
\text { ECMO. }\end{array}$ & $\begin{array}{l}\text { É sugerido que a implantação } \\
\text { antecipada do ECMO evita a } \\
\text { progressão de falha de múltiplos } \\
\text { órgãos, em especial em idade } \\
\text { mais avançada, presença de } \\
\text { comorbidades e estado } \\
\text { imunocomprometido, porém } \\
\text { precisa ser avaliado por conta } \\
\text { das contraindicações. }\end{array}$ \\
\hline 08 & $\begin{array}{l}\text { Remdesivir for the } \\
\text { Treatment of COVID-19- } \\
\text { Final Report. } \\
\text { (BEIGEL } \text { et al., 2020). }\end{array}$ & $\begin{array}{l}\text { Esclarecer se o antiviral } \\
\text { Remdesivir é eficaz para } \\
\text { o tratamento da } \\
\text { COVID-19. }\end{array}$ & $\begin{array}{l}\text { Estudo duplo- } \\
\text { cego } \\
\text { randomizado } \\
\text { unicêntrico }\end{array}$ & $\begin{array}{l}\text { Ensaio controlado por placebo de } \\
\text { remdesivir intravenoso em adultos } \\
\text { hospitalizados com COVID-19 e } \\
\text { com infecção do trato respiratório } \\
\text { inferior. Os pacientes foram } \\
\text { aleatoriamente designados para } \\
\text { receber remdesivir ( } 200 \mathrm{mg} \text { no dia } \\
1 \text { e } 100 \mathrm{mg} \text { diariamente por até } 9 \\
\text { dias) ou placebo por até } 10 \text { dias. } \\
\text { O desfecho primário foi o tempo de } \\
\text { recuperação, definido pela alta } \\
\text { hospitalar ou internação para fins de } \\
\text { controle de infecção. Foram criados } \\
\text { critérios de categorias do } 1 \text { ao } 8 \text {. }\end{array}$ & $\begin{array}{l}\text { Foram submetidos à randomização } 1.062 \\
\text { pacientes, sendo } 541 \text { atribuídos ao } \\
\text { remdesivir e } 521 \text { ao placebo. Para aqueles } \\
\text { que recebem ventilação mecânica (VM) } \\
\text { ou ECMO, a taxa para recuperação foi de } \\
0,98 \text {. Entre os } 285 \text { pacientes que estavam } \\
\text { recebendo VM ou ECMO no momento da } \\
\text { matrícula, os pacientes do grupo } \\
\text { remdesivir receberam essas intervenções } \\
\text { por menos dias do que os do grupo } \\
\text { placebo. A incidência de VM ou ECMO } \\
\text { entre os } 766 \text { pacientes que não estavam } \\
\text { com essas intervenções na matrícula foi } \\
\text { menor no grupo remdesivir do que no } \\
\text { placebo. }\end{array}$ & $\begin{array}{l}\text { Os dados mostram que o } \\
\text { remdesivir foi superior ao } \\
\text { placebo na redução do tempo de } \\
\text { recuperação. Além disso, a VM } \\
\text { e a ECMO se mostraram mais } \\
\text { eficientes no grupo remdesivir, } \\
\text { demonstrando vantagens na } \\
\text { associação. }\end{array}$ \\
\hline
\end{tabular}




\begin{tabular}{|c|c|c|c|c|c|c|}
\hline 09 & $\begin{array}{l}\text { Venous Thromboembolism } \\
\text { Events Following } \\
\text { Venovenous } \\
\text { Extracorporeal Membrane } \\
\text { Oxygenation for Severe } \\
\text { Acute Respiratory } \\
\text { Syndrome Coronavirus } 2 \\
\text { Based on CT Scans. } \\
\text { (PARZY et al., 2020). }\end{array}$ & $\begin{array}{l}\text { Determinar a } \\
\text { prevalência de eventos } \\
\text { de tromboembolismo } \\
\text { venoso em pacientes } \\
\text { infectados com SARA } \\
\text { que requerem ECMO e } \\
\text { comparar eventos de } \\
\text { tromboembolismo } \\
\text { venoso e variáveis de } \\
\text { coagulação em } \\
\text { pacientes que } \\
\text { necessitavam de ECMO } \\
\text { de acordo com o } \\
\text { patógeno. }\end{array}$ & $\begin{array}{l}\text { Observacional } \\
\text { retrospectivo } \\
\text { unicêntrico }\end{array}$ & $\begin{array}{l}\text { Neste estudo, foram incluídos } \\
\text { pacientes internados com SARA } \\
\text { relacionada ao COVID-19 que } \\
\text { necessitam de VV-ECMO. } \\
\text { Também para comparação com } \\
\text { pacientes SARS-CoV-2, foram } \\
\text { extraídos dados de um grupo com } \\
10 \text { pacientes com gripe e um grupo } \\
\text { com } 24 \text { pacientes com pneumonia } \\
\text { adquirida pela comunidade } \\
\text { bacteriana (CAP) à partir de um } \\
\text { banco de dados de um estudo prévio } \\
\text { sobre eventos venosos de } \\
\text { tromboembolismo após VV- } \\
\text { ECMO. }\end{array}$ & $\begin{array}{l}\text { Foram incluídos } 13 \text { pacientes com SARA } \\
\text { que necessitaram de ECMO. Todos esses } \\
\text { pacientes apresentaram embolia venosa } \\
\text { de trombo. }\end{array}$ & 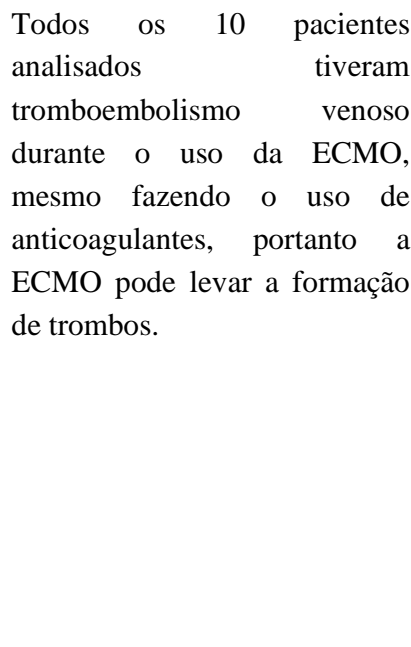 \\
\hline 10 & $\begin{array}{l}\text { Multisystem Inflammatory } \\
\text { Syndrome in Children } \\
\text { Associated with Severe } \\
\text { Acute } 4 \text { Respiratory } \\
\text { Syndrome Coronavirus 2 } \\
\text { Infection (MIS-C): A } \\
\text { Multi-institutional Study } \\
\text { from New York City. } \\
\text { (KAUSHIK et al., 2020). }\end{array}$ & $\begin{array}{l}\text { Avaliar características } \\
\text { clínicas e desfechos da } \\
\text { SARA associada à } \\
\text { MISC-C. }\end{array}$ & $\begin{array}{l}\text { Multicêntrico } \\
\text { observacional } \\
\text { retrospectivo }\end{array}$ & $\begin{array}{l}\text { Estudo de } 33 \text { pacientes pediátricos } \\
\text { (de } 1 \text { mês a } 21 \text { anos) com infecção } \\
\text { confirmada com SARS-CoV-2 que } \\
\text { atendem aos critérios para MIS-C } \\
\text { admitidos de } 23 \text { de abril a } 23 \text { de } \\
\text { maio de } 2020 \text {, a } 3 \text { Hospitais Infantis } \\
\text { de Atenção Terciária de Nova York. }\end{array}$ & $\begin{array}{l}\text { Para o tratamento, a imunoglobulina } \\
\text { intravenosa foi utilizada em } 18 \text { pacientes, } \\
\text { corticosteroides em } 17 \text {, tocilizumabe em } \\
\text { 12, remdesivir em } 7 \text {, vasopressores em } \\
\text { 17, VM em 5, ECMO em } 1 \text { e bomba de } \\
\text { balão intra-aórtico em } 1 \text {. Todos os } \\
\text { pacientes receberam alta domiciliar. O } \\
\text { paciente que estava em ECMO morreu no } \\
6 \text { dia, porque desenvolveu um AVC com } \\
\text { hemorragia subaracnóidea. }\end{array}$ & $\begin{array}{l}\text { Apenas um paciente utilizou da } \\
\text { ECMO e veio à óbito, não } \\
\text { mostrando eficácia neste } \\
\text { estudo. }\end{array}$ \\
\hline 11 & $\begin{array}{l}\text { Anticoagulation } \\
\text { Management in Severe } \\
\text { Coronavirus Disease } 2019 \\
\text { Patients on Extracorporeal } \\
\text { Membrane Oxygenation } \\
\text { (GUO et al., 2020). }\end{array}$ & $\begin{array}{l}\text { Explorar características } \\
\text { especiais de coagulação } \\
\text { e gerenciamento de } \\
\text { anticoagulação em } \\
\text { ECMO em pacientes } \\
\text { com COVID-19. }\end{array}$ & $\begin{array}{c}\text { Observacional } \\
\text { Retrospectivo } \\
\text { unicêntrico }\end{array}$ & $\begin{array}{l}\text { Foi realizado o estudo de } 8 \\
\text { pacientes com COVID-19 que } \\
\text { foram submetidos à VV-ECMO. } \\
\text { Foram coletados os parâmetros } \\
\text { demográficos, comorbidades, } \\
\text { resultados laboratoriais, dados } \\
\text { relacionados ao ECMO e }\end{array}$ & $\begin{array}{l}\text { Oito dos } 667 \text { pacientes confirmados com } \\
\text { COVID- } 19 \text { receberam VV-ECMO, dos } \\
\text { quais } 01 \text { foi desmamado por condição se } \\
\text { deteriorando e veio à óbito. Três outros } \\
\text { morreram de agravamento persistente da } \\
\text { consolidação pulmonar. Outros três } \\
\text { foram desmamados com sucesso e } \\
\text { tiveram alta, e } 01 \text { foi desmamado, porém }\end{array}$ & $\begin{array}{l}\text { Hipercoagulabilidade durante o } \\
\text { suporte ao ECMO em pacientes } \\
\text { com COVID-19 são comuns e } \\
\text { possivelmente aumentam a } \\
\text { propensão para eventos } \\
\text { trombóticos e falha do } \\
\text { oxigenador. Atualmente não há } \\
\text { evidências suficientes para }\end{array}$ \\
\hline
\end{tabular}




\begin{tabular}{|c|c|c|c|c|c|c|}
\hline & & & & $\begin{array}{l}\text { parâmetros de coagulação dos } \\
\text { prontuários. }\end{array}$ & $\begin{array}{l}\text { ainda recebe tratamento de reabilitação. } \\
\text { Dos } 8 \text { pacientes, } 6 \text { desenvolveram lesão } \\
\text { renal aguda e necessitaram de terapia de } \\
\text { substituição renal contínua. }\end{array}$ & $\begin{array}{l}\text { apoiar uma } \text { estratégia de } \\
\text { anticoagulação } \\
\text { Dos } \quad 8 \text { pacientes, } \\
\begin{array}{l}\text { sobreviveram, } \\
\text { considerada uma técnica eficaz. }\end{array}\end{array}$ \\
\hline 12 & $\begin{array}{l}\text { Coronavirus disease } 2019 \\
\text { in patients with inborn } \\
\text { errors of immunity: An } \\
\text { international study } \\
\text { (MEYTS et al., 2020). }\end{array}$ & $\begin{array}{l}\text { Descrever } \\
\text { apresentação, a } \\
\text { manifestações e o } \\
\text { resultado da infecção } \\
\text { por SARS-CoV-2 em } \\
\text { indivíduos com raros } \\
\text { erros de imunidade } \\
\text { (IEI), para informar os } \\
\text { médicos e aumentar a } \\
\text { compreensão da defesa } \\
\text { do hospedeiro contra } \\
\text { SARS-CoV-2. }\end{array}$ & $\begin{array}{l}\text { Observacional } \\
\text { retrospectivo } \\
\text { multicêntrico }\end{array}$ & $\begin{array}{l}\text { Um convite para participar de um } \\
\text { estudo retrospectivo de } 94 \\
\text { pacientes foi distribuído } \\
\text { globalmente às sociedades } \\
\text { científicas e médicas. O } \\
\text { questionário perguntou sobre dados } \\
\text { demográficos, apresentação do } \\
\text { COVID-19, tratamento e desfechos } \\
\text { em pacientes com IEIs e infecção } \\
\text { documentada SARS-CoV-2. }\end{array}$ & $\begin{array}{l}\text { Dez pacientes foram assintomáticos, } 25 \\
\text { foram tratados como ambulatórios, } 28 \\
\text { internações sem tratamento intensivo ou } \\
\text { ventilação, } 13 \text { necessitaram de ventilação } \\
\text { não invasiva ou administração de } \\
\text { oxigênio, } 18 \text { de internação em UTI, } 12 \\
\text { necessitaram de ventilação invasiva e } 3 \\
\text { necessitaram de ECMO, sendo que } 2 \\
\text { morreram. No total nove pacientes (7 } \\
\text { adultos e } 2 \text { crianças) morreram. }\end{array}$ & $\begin{array}{l}\text { Este estudo demonstra que mais } \\
\text { de } 30 \% \text { dos pacientes com IEI } \\
\text { tinham COVID-19 e fatores de } \\
\text { risco predispondo a doença } \\
\text { grave/mortalidade na população } \\
\text { geral também pareciam afetar } \\
\text { pacientes com IEI, incluindo } \\
\text { pacientes mais jovens. O } \\
\text { tratamento com ECMO nesse } \\
\text { caso não foi eficaz, pois dois de } \\
\text { três pacientes vieram à óbito. }\end{array}$ \\
\hline 13 & $\begin{array}{l}\text { Multi-institutional Analysis } \\
\text { of } 100 \text { Consecutive Patients } \\
\text { with COVID-19 and Severe } \\
\text { Pulmonary Compromise } \\
\text { Treatedr with } \\
\text { Extracorporeal Membrane } \\
\text { Oxygenation: Outcomes } \\
\text { and Trends Over Time } \\
\\
\text { (JACOBS et al., 2021). }\end{array}$ & $\begin{array}{l}\text { Rever uma experiência } \\
\text { clínica em pacientes } \\
\text { com } \quad \text { COVID-19 } \\
\text { apoiados com ECMO. }\end{array}$ & $\begin{array}{l}\text { Multicêntrico } \\
\text { prospectivo de } \\
\text { coorte }\end{array}$ & $\begin{array}{l}\text { Uma experiência clínica em } 100 \\
\text { pacientes consagrados, em } 20 \\
\text { hospitais, com COVID-19 apoiados } \\
\text { com ECMO. Os dados capturados } \\
\text { incluíram características do } \\
\text { paciente, fatores de risco pré- } \\
\text { COVID-19, comorbidades e } \\
\text { confirmação do diagnóstico } \\
\text { COVID-19, características de } \\
\text { suporte ao ECMO, medicamentos } \\
\text { específicos utilizados na tentativa } \\
\text { de tratar o COVID-19 e desfechos } \\
\text { de curto prazo por meio da alta } \\
\text { hospitalar. }\end{array}$ & $\begin{array}{l}\text { Todos os } 100 \text { pacientes foram separados } \\
\text { da ECMO: } 50 \text { pacientes sobreviveram e } \\
50 \text { pacientes morreram. A taxa de } \\
\text { sobrevivência com VV-ECMO foi de } \\
51 \% \text {, enquanto com VA-ECMO foi de } \\
25 \% \text {. Dos } 50 \text { sobreviventes, } 49 \text { receberam } \\
\text { alta do hospital e } 1 \text { permaneceu internado. } \\
\text { Os sobreviventes eram geralmente mais } \\
\text { jovens. }\end{array}$ & $\begin{array}{l}\text { A ECMO facilita o resgate e a } \\
\text { sobrevivência de pacientes em } \\
\text { estado crítico com COVID-19. } \\
\text { A sobrevivência de pacientes } \\
\text { apoiados apenas com VV- } \\
\text { ECMO é de } 51 \% \text {. Existe uma } \\
\text { variação substancial no } \\
\text { tratamento medicamentoso da } \\
\text { COVID-19, mas a ECMO } \\
\text { oferece uma estratégia de } \\
\text { resgate razoável. }\end{array}$ \\
\hline
\end{tabular}




\begin{tabular}{|c|c|c|c|c|c|c|}
\hline 14 & $\begin{array}{l}\text { Characteristics of mortal } \\
\text { COVID-19 cases compared } \\
\text { to the survivors } \\
\text { (ZHOU et al., 2020). }\end{array}$ & $\begin{array}{l}\text { Explorar fatores de risco } \\
\text { associados aos óbitos do } \\
\text { COVID-19. }\end{array}$ & $\begin{array}{l}\text { Revisão } \\
\text { sistemática }\end{array}$ & $\begin{array}{l}\text { Foram pesquisados artigos no } \\
\text { PubMed, EMBASE e CNKI e à } \\
\text { partir disso foram realizadas } \\
\text { análises. Dezenove estudos foram } \\
\text { utilizados. }\end{array}$ & $\begin{array}{l}\text { A VM e a ECMO desempenham papel } \\
\text { crucial no tratamento de casos críticos, } \\
\text { embora possam levar a infecções } \\
\text { subsequentes, pois as bactérias podem } \\
\text { invadir os pulmões através do tubo de } \\
\text { traqueostomia, podendo causar danos } \\
\text { pulmonares, pneumotórax e a } \\
\text { incapacidade de interromper o suporte do } \\
\text { ventilador. }\end{array}$ & $\begin{array}{l}\text { A VM e ECMO desempenham } \\
\text { excelente papel no tratamento } \\
\text { de casos críticos, embora seja } \\
\text { importante determinar a } \\
\text { condição geral dos pacientes } \\
\text { infectados e reduzir a taxa de } \\
\text { mortalidade. }\end{array}$ \\
\hline 15 & $\begin{array}{l}\text { Intensive care admissions } \\
\text { of children with paediatric } \\
\text { inflammatory multisystem } \\
\text { syndrome temporally } \\
\text { associated with SARS- } \\
\text { CoV-2 (PIMS-TS) in the } \\
\text { UK: a multicentre } \\
\text { observational study. } \\
\text { (DAVIES et al., 2020). }\end{array}$ & $\begin{array}{l}\text { Descrever as } \\
\text { características clínicas, } \\
\text { curso, manejo e } \\
\text { desfechos de pacientes } \\
\text { admitidos em internação } \\
\text { na UTI pediátrica } \\
\text { (PICUs) em pacientes } \\
\text { pediátricos com MIC-C } \\
\text { associada ao SARS- } \\
\text { CoV-2. }\end{array}$ & $\begin{array}{l}\text { Observacional } \\
\text { multicêntrico } \\
\text { retrospectivo }\end{array}$ & $\begin{array}{l}\text { Foi realizado um estudo em } \\
\text { crianças, admitidas no PICUs no } \\
\text { Reino Unido. Foram analisados } \\
\text { dados coletados rotineiramente, } \\
\text { incluindo detalhes demográficos, } \\
\text { apresentandor características } \\
\text { clínicas, comorbidades subjacentes, } \\
\text { marcadores laboratoriais, achados } \\
\text { ecocardiográficos, intervenções, } \\
\text { tratamentos e desfechos. }\end{array}$ & $\begin{array}{l}\text { Três crianças precisaram de ECMO, } \\
\text { sendo que duas morreram. }\end{array}$ & $\begin{array}{l}\text { Aneurismas da artéria coronária } \\
\text { parecem ser uma complicação } \\
\text { importante nos pacientes com a } \\
\text { COVID-19. A ECMO nesse } \\
\text { caso não foi eficaz por sua } \\
\text { baixa taxa de sobrevivência. }\end{array}$ \\
\hline 16 & $\begin{array}{l}\text { Clinical care of pregnant } \\
\text { and postpartum women } \\
\text { with COVID-19: Living } \\
\text { recommendations from the } \\
\text { National } \quad \text { COVID-19 } \\
\text { Clinical } \\
\text { Taskforce } \\
\text { (VOGEL } \text { et al., 2020). }\end{array}$ & $\begin{array}{l}\text { Descrever } \\
\text { recomendações }\end{array}$ & $\begin{array}{c}\text { Revisão } \\
\text { sistemática }\end{array}$ & $\begin{array}{l}\text { Avaliação e síntese de evidências } \\
\text { para informar o desenvolvimento } \\
\text { dessas recomendações. O estudo } \\
\text { inclui } 13 \text { especialistas da saúde. } \\
\text { Foram incluídos na pesquisa o } \\
\text { remdesivir, hidroxicloroquina, } \\
\text { tromboembolismo venoso e a } \\
\text { ECMO. }\end{array}$ & $\begin{array}{l}\text { Não há atualmente evidência primária } \\
\text { sobre o uso da ECMO e seus benefícios e } \\
\text { danos, embora se saiba ser eficaz em } \\
\text { alguns grupos de pacientes adultos com } \\
\text { doenças críticas. A Força Tarefa } \\
\text { recomenda que o posicionamento } \\
\text { propenso seja considerado em mulheres } \\
\text { que recebem qualquer forma de } \\
\text { oxigenoterapia suplementar ou mulheres } \\
\text { em VM, embora deva-se tomar cuidado. }\end{array}$ & $\begin{array}{l}\text { O encaminhamento à um centro } \\
\text { de ECMO pode ser considerado } \\
\text { para gestantes em VM, embora } \\
\text { essa decisão precise considerar } \\
\text { idade gestacional, viabilidade } \\
\text { fetal, bem-estar fetal e os riscos } \\
\text { e benefícios para a mulher e o } \\
\text { bebê. }\end{array}$ \\
\hline 17 & $\begin{array}{lrr}\text { Global } & \text { Impact } & \text { of } \\
\text { Coronavirus } & \text { Disease } & 2019\end{array}$ & $\begin{array}{l}\text { Fornecer avaliação } \\
\text { contemporânea e global } \\
\text { da estimativa de morte e }\end{array}$ & $\begin{array}{c}\text { Revisão } \\
\text { sistemática meta } \\
\text { análise }\end{array}$ & $\begin{array}{l}\text { Medline, Embase e a biblioteca } \\
\text { Cochrane foram usados como fonte } \\
\text { de pesquisa. Foram usados estudos }\end{array}$ & $\begin{array}{l}\text { A VM foi necessária em } 67,7 \% \text { de casos, } \\
\text { suporte vasopressor em } 65,9 \% \text {, terapia } \\
\text { renal de reposição em } 16,9 \% \text { e ECMO em }\end{array}$ & $\begin{array}{l}\text { Pacientes gravemente doentes } \\
\text { com COVID-19 que estão } \\
\text { internados na UTI requerem }\end{array}$ \\
\hline
\end{tabular}




\begin{tabular}{|c|c|c|c|c|c|c|}
\hline & $\begin{array}{l}\text { Infection Requiring } \\
\text { Admission to the ICU } \\
\text { (TAN et al., 2020). }\end{array}$ & $\begin{array}{l}\text { fatores de risco para } \\
\text { doenças graves em } \\
\text { pacientes internados em } \\
\text { UTI com COVID-19. }\end{array}$ & & $\begin{array}{l}\text { que relataram taxa de mortalidade } \\
\text { hospitalar de pacientes adultos com } \\
\text { COVID-19, internados em UTI } \\
\text { atenderam aos critérios de } \\
\text { elegibilidade do estudo. }\end{array}$ & $\begin{array}{l}6,4 \% \text { dos casos, onde sua taxa de } \\
\text { mortalidade foi de } 33 \% .\end{array}$ & $\begin{array}{l}\text { apoio substancial dos órgãos e } \\
\text { cuidados prolongados de UTI e } \\
\text { nível hospitalar. Neste caso o } \\
\text { uso da ECMO foi eficaz, tendo } \\
\text { apenas 33\% de mortalidade. }\end{array}$ \\
\hline 18 & $\begin{array}{l}\text { Prone positioning for } \\
\text { patients intubated for } \\
\text { severe acute respiratory } \\
\text { distress syndrome (ARDS) } \\
\text { secondary to COVID-19: a } \\
\text { retrospective observational } \\
\text { cohort study } \\
\text { (WEISS et al 2020). }\end{array}$ & $\begin{array}{l}\text { Investigar o efeito do } \\
\text { posicionamento } \\
\text { propenso para pacientes } \\
\text { com SARA secundário } \\
\text { ao COVID-19 que } \\
\text { necessitavam de VM. }\end{array}$ & $\begin{array}{l}\text { Retrospectivo de } \\
\text { coorte } \\
\text { observacional } \\
\text { unicêntrico }\end{array}$ & $\begin{array}{l}\text { Foi realizado um estudo de } \\
\text { pacientes entubados em estado } \\
\text { crítico com COVID-19. Os } \\
\text { pacientes foram acompanhados até } \\
\text { a alta hospitalar. }\end{array}$ & $\begin{array}{l}\text { Um total de } 74 \% \text { sujeitos sobreviveram à } \\
\text { alta, com } 05 \text { necessitando de ECMO e os } \\
26 \% \text { restantes morreram. Os que tiveram } \\
\text { alta hospitalar e apresentaram maior } \\
\text { probabilidade de ter uma melhora na } \\
\text { relação PA } 2 / \mathrm{Fi} 2 \geq 20 \% \text {, em comparação } \\
\text { com aqueles que necessitavam de ECMO } \\
\text { ou que morreram. }\end{array}$ & $\begin{array}{l}\text { A ECMO foi considerada se a } \\
\text { oxigenação não poderia ser } \\
\text { mantida sob ventilação } \\
\text { protetora pulmonar com } \\
\text { posicionamento propenso, } \\
\text { paralisia e vasodilatadores } \\
\text { pulmonares inalados. O. Os } \\
\text { cinco pacientes que utilizaram a } \\
\text { ECMO sobreviveram, } \\
\text { sugerindo eficácia. }\end{array}$ \\
\hline 19 & $\begin{array}{l}\text { The Use of Venovenous } \\
\text { Extracorporeal Membrane } \\
\text { Oxygenation in COVID-19 } \\
\text { Infection: One Region's } \\
\text { Comprehensive Experience } \\
\text { (BERGMAN et al., 2021). }\end{array}$ & $\begin{array}{l}\text { Avaliar o uso de V-V } \\
\text { ECMO e os desfechos } \\
\text { clínicos durante o } \\
\text { primeiro surto de casos } \\
\text { de SARA grave } \\
\text { associados ao COVID- } \\
19 .\end{array}$ & $\begin{array}{l}\text { Retrospectivo } \\
\text { observacional } \\
\text { multicêntrico }\end{array}$ & $\begin{array}{l}\text { Foi feito um estudo incluindo } 35 \\
\text { pacientes adultos colocados em V- } \\
\text { V ECMO devido a SARA. Houve } \\
\text { infusão de heparina durante a } \\
\text { canulação e continuou durante a } \\
\text { duração da ECMO, à menos que o } \\
\text { paciente desenvolvesse uma } \\
\text { contraindicação ao anticoagulante } \\
\text { sistêmico. }\end{array}$ & $\begin{array}{l}\text { Vinte seis dos } 35 \text { pacientes suportados } \\
\text { com V-V ECMO sobreviveram à } \\
\text { descanulação pós-ECMO de } 60 \text { dias; e } 9 \\
\text { morreram. }\end{array}$ & $\begin{array}{l}\text { O suporte ECMO precoce, em } \\
\text { comparação com o manejo } \\
\text { convencional, relhora } \\
\text { significativamente a sobrevida. } \\
\text { As complicações do circuito } \\
\text { ECMO foram trombose, } \\
\text { insuficiencia renal e infecção } \\
\text { secundária. Nesta caso a ECMO } \\
\text { foi eficaz. }\end{array}$ \\
\hline 20 & $\begin{array}{l}\text { Clinical Findings and } \\
\text { Disease Severity in } \\
\text { Hospitalized Pregnant } \\
\text { Women With Coronavirus } \\
\text { Disease 2019. }\end{array}$ & 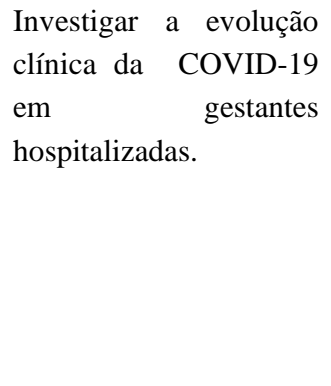 & $\begin{array}{l}\text { Prospectivo de } \\
\text { coorte } \\
\text { multicêntrico }\end{array}$ & $\begin{array}{l}\text { Foi feita uma avaliação de } 77 \\
\text { mulheres grávidas internadas com a } \\
\text { infecção SARA-CoV-2 em } 12 \\
\text { maternidades diferentes na Itália. } \\
\text { Foram coletados dados sobre } \\
\text { tratamentos COVID-19, evolução } \\
\text { clínica durante a gravidez, }\end{array}$ & $\begin{array}{l}\text { Das } 77 \text { pacientes, } 14 \text { apresentavam } \\
\text { doença grave (parto urgente). Dois terços } \\
\text { dos pacientes foram internados durante } \\
\text { o } 3 \text { trimestre, e } 84 \% \text { foram sintomáticos } \\
\text { na internação. Onze pacientes foram } \\
\text { submetidos ao parto urgente e seis } \\
\text { deram entrada na UTI. Uma mulher com } \\
\text { a doença mais grave teve seu parto } \\
\text { prematuro e utilizou da ECMO, com }\end{array}$ & $\begin{array}{l}\text { Foi detectada melhora } \\
\text { significativa pós-parto em } \\
\text { condições clínicas maternas } \\
\text { em seis pacientes com doença } \\
\text { grave. Destas } 6 \text { pacientes, uma } \\
\text { estava utilizando a terapia } \\
\text { ECMO, a qual foi eficaz. }\end{array}$ \\
\hline
\end{tabular}




\begin{tabular}{|c|c|c|c|c|c|c|}
\hline & & & & $\begin{array}{l}\text { necessidade de ECMO e internação } \\
\text { em UTI. }\end{array}$ & $\begin{array}{l}\text { posterior melhora nas condições clínicas; } \\
\text { não houve mortes. }\end{array}$ & \\
\hline 21 & $\begin{array}{l}\text { Mechanical ventilation and } \\
\text { mortality among } 223 \\
\text { critically ill patients with } \\
\text { coronavirus disease 2019: } \\
\text { A multicentric study in } \\
\text { Germany } \\
\text { (ROEDL et al., 2020). }\end{array}$ & 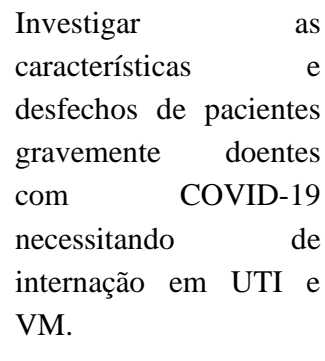 & $\begin{array}{c}\text { Multicentrico de } \\
\text { coorte } \\
\text { observacional } \\
\text { retrospectivo }\end{array}$ & $\begin{array}{l}\text { Foram incluídos } 223 \text { pacientes } \\
\text { adultos (maiores de } 18 \text { anos) em } \\
\text { estado crítico com COVID-19 que } \\
\text { completaram a UTI entre fevereiro } \\
\text { e junho de } 2020 .\end{array}$ & $\begin{array}{l}\text { No total, } 75 \% \text { dos pacientes precisavam } \\
\text { de VM. A ECMO foi necessária em } 20 \\
\text { pacientes que evoluíram para SARA } \\
\text { grave. A mortalidade da UTI foi de } 35 \% \text {, } \\
\text { sendo } 44 \% \text { entre pacientesem VM, } 7 \% \\
\text { em pacientes que nào usaram VM. Dos } 20 \\
\text { pacientes, } 13 \text { dos que usaram ECMO } \\
\text { morreram. }\end{array}$ & $\begin{array}{l}\text { Tanto a idade mais avançada } \\
\text { quanto as comorbidades } \\
\text { estiveram associadas à } \\
\text { mortalidade. A ECMO foi } \\
\text { associada a mais casos de } \\
\text { mortalidade do que melhora. }\end{array}$ \\
\hline 22 & $\begin{array}{l}\text { Right ventricular } \\
\text { dysfunction in critically ill } \\
\text { COVID-19 ARDS } \\
\text { (BLEAKLEY et al., 2020). }\end{array}$ & $\begin{array}{lr}\text { Detalhar o fenótipo } \\
\text { específico e associações } \\
\text { clínicas } & \text { de } \\
\text { comprometimento } & \text { do } \\
\text { ventrículo direito } & \text { na } \\
\text { SARA associada } & \text { ao } \\
\text { COVID-19. } & \end{array}$ & $\begin{array}{l}\text { Observacional } \\
\text { retrospectivo } \\
\text { unicêntrico }\end{array}$ & $\begin{array}{l}\text { Medidas de ecocardiografia } \\
\text { transtorácica (TTE) da função } \\
\text { ventrículo direito foram coletadas } \\
\text { em } 90 \text { pacientes (em média de } 52 \mathrm{e} \\
10 \text { anos de idade) em VM e } \\
\text { criticamente indispostos para } \\
\text { associações com dados clínicos, } \\
\text { ventilatórios e laboratoriais. } 42 \% \\
\text { dos pacientes usaram VV- ECMO. }\end{array}$ & $\begin{array}{l}\text { Anormalidades de ventrículo direito } \\
\text { foram mais comuns em formas mais } \\
\text { graves de COVID-19. Os fatores de } \\
\text { coagulopatia estão relacionados ao uso da } \\
\text { ECMO. }\end{array}$ & $\begin{array}{l}\text { A COVID-19 está associada à } \\
\text { um fenótipo específico de } \\
\text { prejuízo do ventrículo direito. } \\
\text { A coagulopatia foi influenciada } \\
\text { por uma ampla gama de } \\
\text { fatores, incluindo VV- ECMO. } \\
\text { O uso da ECMO neste estudo } \\
\text { não obteve dados concretos. }\end{array}$ \\
\hline 23 & $\begin{array}{l}\text { Critically ill patients with } \\
\text { COVID-19 with ECMO } \\
\text { and artificial liver plasma } \\
\text { Exchange } \\
\text { (LIU et al., 2020). }\end{array}$ & $\begin{array}{l}\text { Descrever } \\
\text { epidemiologia, } \\
\text { características clínicas e } \\
\text { preditores de desfechos } \\
\text { de pacientes COVID-19 } \\
\text { graves que necessitam } \\
\text { de VM e ECMO. }\end{array}$ & $\begin{array}{l}\text { Retrospectivo } \\
\text { unicêntrico } \\
\text { observacional }\end{array}$ & $\begin{array}{l}\text { Vinte e três pacientes em estado } \\
\text { grave da COVID- } 19 \text {, com em média } \\
66 \text { anos foram submetidos à } \\
\text { internação na UTI, MV, troca } \\
\text { artificial de plasma do fígado e } \\
\text { ECMO (em média de } 22 \text { dias). }\end{array}$ & $\begin{array}{l}\text { Nove pacientes necessitavam de } \\
\text { oxigênio de alto fluxo, 61\% dos } \\
\text { pacientes receberam VM, 26\% } \\
\text { necessitaram de ECMO e } 43 \% \text { receberam } \\
\text { troca artificial de plasma de fígado. A } \\
\text { mortalidade foi } 0 \text {. A relação } \mathrm{PaO} 2 / \mathrm{FiO} 2 \\
\text { (medida de oxigenacão usada para } \\
\text { classificar a SARA em leve, moderada e } \\
\text { grave) foi muito melhorada após o } \\
\text { ECMO. }\end{array}$ & $\begin{array}{l}\text { A ECMO reduziu os requisitos } \\
\text { para suporte ao ventilador e } \\
\text { ajudou a ampliar o tempo de } \\
\text { sobrevivência dos pacientes e } \\
\text { aumentar a chance de } \\
\text { tratamentos. As comorbidades } \\
\text { mais comuns foram diabetes e } \\
\text { hipertensão. }\end{array}$ \\
\hline
\end{tabular}




\begin{tabular}{|c|c|c|c|c|c|c|}
\hline 24 & $\begin{array}{l}\text { Extracorporeal membrane } \\
\text { oxygenation in patients } \\
\text { with severe respiratory } \\
\text { failure from COVID-19. } \\
\text { (SHAEFI et al., 2021). }\end{array}$ & $\begin{array}{l}\text { Avaliar } \\
\text { características clínicas e } \\
\text { os desfechos de } 190 \\
\text { pacientes tratados com } \\
\text { ECMO dentro de 14 dias } \\
\text { após a internação na } \\
\text { UTI, }\end{array}$ & $\begin{array}{l}\text { Multicêntrico de } \\
\text { coorte } \\
\text { prospectivo }\end{array}$ & $\begin{array}{l}\text { Foi realizado um teste-alvo de } \\
\text { recebimento de ECMO versus } \\
\text { nenhum recebimento de ECMO no } \\
\text { prazo de } 7 \text { dias após a internação da } \\
\text { UTI. }\end{array}$ & $\begin{array}{l}\text { Dos } 190 \text { que usaram ECMO, } 33,2 \% \\
\text { morreram, } 49,5 \% \text { tiveram alta e } 17,4 \% \\
\text { ainda estavam internados no dia } 60 ; 553 \\
\text { dos } 1167 \text { que não usaram ECMO } \\
\text { morreram. As complicações mais comuns } \\
\text { após a canulação do ECMO foram } \\
\text { pneumonia bacteriana }(34,7 \%), \\
\text { sangramento (27,9\%), eventos } \\
\text { trombóticos }(22,6 \%) \text { e lesão renal aguda } \\
\text { que necessitam de terapia de reposição } \\
\text { renal }(21,8 \%) .\end{array}$ & $\begin{array}{l}\text { A ECMO pode reduzir a } \\
\text { mortalidade de pacientes com a } \\
\text { COVID- } 19 .\end{array}$ \\
\hline 25 & $\begin{array}{l}\text { Adult cardiovascular } \\
\text { surgery and the coronavirus } \\
\text { disease } 2019 \text { (COVID-19) } \\
\text { pandemic: the Italian } \\
\text { experience. } \\
\text { (DONATELLI et al., } \\
\text { 2020). }\end{array}$ & $\begin{array}{l}\text { Relatar a experiência } \\
\text { italiana durante a } \\
\text { pandemia da COVID-19 } \\
\text { no cenário da cirurgia } \\
\text { cardiovascular. }\end{array}$ & $\begin{array}{c}\text { Revisão } \\
\text { sistemática }\end{array}$ & $\begin{array}{l}\text { Foi feita uma avaliação da gestão de } \\
\text { hospitais italianos durante a } \\
\text { pandemia, principalmente referente } \\
\text { às cirurgias cardiovasculares. }\end{array}$ & $\begin{array}{l}\text { Pacientes mais jovens e com menos } \\
\text { comorbidades são prioridade na ECMO, } \\
\text { portanto seu uso precoce ajuda a salvar } \\
\text { vidas. Pacientes com idade avançada, } \\
\text { comorbidades ou falha multiplos órgãos } \\
\text { são difíceis de gerenciar. No geral, } \\
\text { pacientes mais jovens com comorbidades } \\
\text { menores ou sem comorbidades são a } \\
\text { maior prioridade clínica. }\end{array}$ & $\begin{array}{l}\text { Constatou-se que a COVID-19 } \\
\text { aumenta os problemas } \\
\text { cardiovasculares, precisando } \\
\text { assim de mais cirurgias. A } \\
\text { ECMO por sua vez, acaba por } \\
\text { diminuir os riscos causados pelo } \\
\text { vírus. }\end{array}$ \\
\hline 26 & 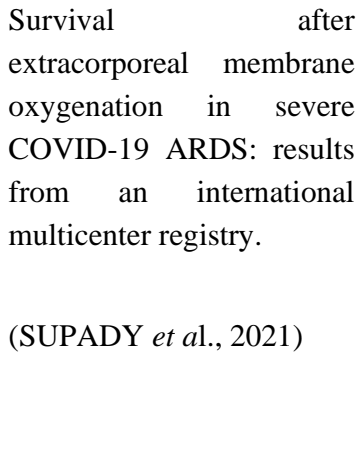 & $\begin{array}{l}\text { Avaliar os resultados de } \\
\text { pacientes com COVID- } \\
19 \text { submetidos à ECMO. }\end{array}$ & $\begin{array}{l}\text { Multicêntrico } \\
\text { retrospectivo de } \\
\text { coorte }\end{array}$ & $\begin{array}{l}\text { Foram analisados } 127 \text { pacientes } \\
\text { após a implantação da ECMO. }\end{array}$ & $\begin{array}{l}\text { Maior sobrevida foi observada em } \\
\text { pacientes menores de } 71 \text { anos. Os } \\
\text { pacientes que estavam em VM antes da } \\
\text { ECMO por menos de } 7 \text { dias } \\
\text { apresentaram taxa de sobrevivência } \\
\text { ligeiramente maior do que aqueles com } \\
\text { curso de VM mais longo. A taxa de } \\
\text { sobrevida em pacientes com ECMO } \\
\text { precoce foi maior. }\end{array}$ & $\begin{array}{l}\text { O uso de V-V ECMO em } \\
\text { SARA associada ao COVID- } \\
\text { 19, também após períodos } \\
\text { prolongados de VM em } \\
\text { pacientes selecionados acabou } \\
\text { por ajudar em resultados } \\
\text { positivos destes pacientes. Os } \\
\text { limites de idade superior devem } \\
\text { ser vistos com cautela e não } \\
\text { tomados como a única razão } \\
\text { para reter o tratamento ECMO. }\end{array}$ \\
\hline
\end{tabular}




\begin{tabular}{|c|c|c|c|c|c|c|}
\hline 27 & $\begin{array}{l}\text { Nebulized in-line } \\
\text { endotracheal dornase alfa } \\
\text { and albuterol administered } \\
\text { to mechanically ventilated } \\
\text { COVID-19 patients: a case } \\
\text { series. } \\
\text { (WEBER et al., 2020) }\end{array}$ & $\begin{array}{l}\text { Avaliar o efeito do } \\
\text { medicamento Dornase } \\
\text { alfa em pacientes com } \\
\text { COVID-19. }\end{array}$ & $\begin{array}{l}\text { Unicêntrico } \\
\text { observacional } \\
\text { retrospectivos }\end{array}$ & $\begin{array}{l}\text { Dados demográficos e clinicos } \\
\text { foram coletados à partir dos } \\
\text { prontuários eletrônicos de cinco } \\
\text { pacientes em VM com COVID-19, } \\
\text { incluindo três necessitando de } \\
\text { ECMO e administrados com } \\
\text { dornase alfa. }\end{array}$ & $\begin{array}{l}\text { A fração dos requisitos de oxigênio } \\
\text { inspirados foi reduzida para todos os } \\
\text { cinco pacientes após o início da } \\
\text { administração dornase alfa. Todos os } 3 \\
\text { pacientes foram extubados com sucesso } \\
\text { da ECMO, tiveram alta hospitalar e } \\
\text { permanecem vivos. }\end{array}$ & $\begin{array}{l}\text { Os resultados sugerem que } \\
\text { dornase alfa é bem tolerada } \\
\text { por pacientes com COVID19 } \\
\text { grave. Todos os pacientes que } \\
\text { utilizaram a ECMO tiveram } \\
\text { sucesso. }\end{array}$ \\
\hline 28 & $\begin{array}{l}\text { Severe respiratory failure in } \\
\text { the course of coronavirus } \\
\text { disease } 2019 \text { treated with } \\
\text { extracorporeal membrane } \\
\text { oxygenation. } \\
\text { (SUWALSK et al., 2020), }\end{array}$ & $\begin{array}{lll}\text { Avaliar a } & \text { ECMO no } \\
\text { curso da } & \text { COVID-19 } \\
2019 . & & \end{array}$ & $\begin{array}{c}\text { Revisão } \\
\text { sistemática }\end{array}$ & $\begin{array}{l}\text { Neste estudo, foi relatado o caso de } \\
\text { um homem de } 36 \text { anos que foi } \\
\text { tratado com ECMO. Uma busca } \\
\text { sistemática para artigos sobre o uso } \\
\text { de ECMO em pacientes com } \\
\text { COVID-19 foi realizada no } \\
\text { PubMed em } 19 \text { de abril de } 2020 . \\
\text { Apenas estudos que relataram o } \\
\text { número de mortes foram incluídos. }\end{array}$ & $\begin{array}{l}\text { Devido a maior deterioração respiratória } \\
\text { e agravamento da condição, foi tomada a } \\
\text { decisão de colocar o paciente em ECMO } \\
\text { no dia 8. A VV-ECMO foi instituída } \\
\text { cutâneamente após o ultrassom à beira do } \\
\text { leito e a examinação dos vasos femorais. } \\
\text { Infelizmente a doença piorou e o paciente } \\
\text { morreu de pneumonia grave, choque } \\
\text { séptico, respiração e falência no } 12^{\circ} \text { dia de } \\
\text { hospitalização. }\end{array}$ & $\begin{array}{l}\text { A taxa de sobrevivência foi } \\
\text { muito alta para infecções } \\
\text { adquiridas na comunidade em } \\
\text { relação aos outros artigos } \\
\text { estudados, porém neste caso a } \\
\text { ECMO foi ineficaz, havendo } \\
\text { morte do paciente, } \\
\text { provavelmente por conta de } \\
\text { suas comorbidades. }\end{array}$ \\
\hline 29 & $\begin{array}{l}\text { Oropharyngeal hemorrhage } \\
\text { in patients with COVID-19: } \\
\text { A multiinstitutional case } \\
\text { series. } \\
\text { (MULCAHY et al., 2020) }\end{array}$ & $\begin{array}{l}\text { Observar a hemorragia } \\
\text { orofaríngea em } \\
\text { pacientes com COVID- } \\
19 \text { entubados. }\end{array}$ & $\begin{array}{l}\text { Multicêntrico de } \\
\text { coorte } \\
\text { observacional } \\
\text { retrospectivo }\end{array}$ & $\begin{array}{l}\begin{array}{l}\text { Foi relatado cinco casos de } \\
\text { hemorragia orofaríngea em }\end{array} \\
\text { pacientes COVID-19, entubados } \\
\text { em VM. }\end{array}$ & $\begin{array}{l}\text { Para pacientes que necessitam de } \\
\text { ECMO, complicações hemorrágicas } \\
\text { ocorrem em até } 45 \% \text { dos pacientes e } \\
\text { a incidência de hemorragia orofaria é de } \\
\text { até } 30 \% \text {, devido ao uso de } \\
\text { anticoagulantes. }\end{array}$ & $\begin{array}{l}\text { Os pacientes entubados por } \\
\text { longos períodos de tempo } \\
\text { podem estar em maior risco } \\
\text { de lesão por ETT. Devido ao } \\
\text { uso de anticoagulantes, } \\
\text { pacientes em ECMO tendem a } \\
\text { ter uma maior hemorragia. }\end{array}$ \\
\hline 30 & $\begin{array}{l}\text { Extracorporeal Membrane } \\
\text { Oxygenation Therapy for } \\
\text { Critically Ill Coronavirus } \\
\text { Disease } 2019 \text { Patients in } \\
\text { Wuhan, China: A } \\
\text { Retrospective Multicenter } \\
\text { Cohort Study. }\end{array}$ & $\begin{array}{l}\text { Acompanhar pacientes } \\
\text { com COVID-19 que } \\
\text { precisam do uso da } \\
\text { terapia ECMO. }\end{array}$ & $\begin{array}{l}\text { Retrospectivo de } \\
\text { coorte } \\
\text { multicêntrico }\end{array}$ & $\begin{array}{l}\text { Foram analisados } 88 \text { pacientes } \\
\text { para identificar os fatores de risco } \\
\text { associados ao desmame da ECMO } \\
\text { mal sucedida. Houve a comparação } \\
\text { entre pacientes que receberam VV- } \\
\text { ECMO com aqueles que receberam } \\
\text { VM. }\end{array}$ & $\begin{array}{l}\text { Dos } 88 \text { pacientes que receberam terapia } \\
\text { ECMO, } 27 \text { foram desmamados com } \\
\text { sucesso da ECMO e } 61 \text { sem sucesso. } \\
\text { Além disso, } 15,15 \text { e } 65 \text { pacientes foram } \\
\text { desmamados do VM, liberados do } \\
\text { hospital ou morreram durante a } \\
\text { internação, respectivamente. }\end{array}$ & $\begin{array}{l}\text { Llinfocitopenia, dímero-D mais } \\
\text { alto e hipercapnia levam a } \\
\text { resultado ruim do uso de } \\
\text { ECMO. Traqueostomia poderia } \\
\text { facilitar o desmame da ECMO. } \\
\text { O uso da MV facilitou o }\end{array}$ \\
\hline
\end{tabular}




\begin{tabular}{|c|c|c|c|c|c|c|}
\hline & (FANG et al., 2021). & & & & & $\begin{array}{l}\text { desmame. Neste caso a ECMO } \\
\text { foi pouco eficaz. }\end{array}$ \\
\hline 31 & $\begin{array}{l}\text { Factors associated with } \\
\text { clinical outcomes in } \\
\text { patients with Coronavirus } \\
\text { Disease } 2019 \text { in } \\
\text { Guangzhou, China. } \\
\text { (LEI et al., 2020) }\end{array}$ & $\begin{array}{lr}\text { Descrever } & \text { as } \\
\text { características clínicas e } & \text { fatores } \\
\text { explorar } & \text { e } \\
\text { virológicos } & \\
\text { imunológicos } & \\
\text { associados } & \text { aos } \\
\text { desfechos de } & \text { pacientes } \\
\text { hospitalizados } & \text { com } \\
\text { COVID-19. }\end{array}$ & $\begin{array}{c}\text { Retrospectivo } \\
\text { observacional } \\
\text { unicentrico }\end{array}$ & $\begin{array}{l}\text { Duzentos e noventa e sete pacientes } \\
\text { com COVID-19 internados foram } \\
\text { analisados durante um período } \\
\text { médio de } 17 \text { dias. }\end{array}$ & $\begin{array}{l}\text { Os pacientes desenvolveram } \\
\text { insuficiência respiratória e foram } \\
\text { submetidos à VM. A ECMO foi } \\
\text { realizada em } 4(1,3 \%) \text { dos pacientes. Um } \\
(0,3 \%) \text { paciente do sexo feminino com } \\
\text { idade de } 30 \text { anos recebeu uma operação } \\
\text { bem sucedida por causa de cisto ovariano } \\
\text { rompido e um (0,3\%) paciente de } 82 \text { anos } \\
\text { morreu de falha múltipla de órgãos } \\
\text { mesmo recebendo tratamento ECMO. }\end{array}$ & $\begin{array}{l}\text { O esquema de terapia ECMO } \\
\text { poderia tratar com sucesso o } \\
\text { COVID-19, mesmo sem } \\
\text { regimes antivirais eficazes } \\
\text { atualmente. }\end{array}$ \\
\hline 32 & $\begin{array}{l}\text { Pharmacologic Treatments } \\
\text { and Supportive Care for } \\
\text { Middle East Respiratory } \\
\text { Syndrome. } \\
\text { (KLAIN et al., 2020) }\end{array}$ & $\begin{array}{l}\text { Desenvolver um } \\
\text { protocolo através de } \\
\text { pesquisas de dados para } \\
\text { estudos que descrevem } \\
\text { o tratamento de } \\
\text { sindrome respiratória do } \\
\text { Oriente Médio (MERS) } \\
\text { e óbitos em pacientes do } \\
\text { MERS. }\end{array}$ & $\begin{array}{c}\text { Revisão } \\
\text { sistemática e } \\
\text { meta analises }\end{array}$ & $\begin{array}{l}\text { Foi realizado uma revisão } \\
\text { sistemática para resumir a base de } \\
\text { evidências atuais para o tratamento } \\
\text { do MERS, incluindo tratamentos } \\
\text { específicos contra o MERS, } \\
\text { terapias farmacológicas adjuntivas } \\
\text { e cuidados de apoio. }\end{array}$ & $\begin{array}{l}\text { Um estudo, mostrou taxas de } \\
\text { mortalidade reduzidas em pacientes } \\
\text { graves de MERS com ECMO; nenhum } \\
\text { outro estudo mostrou um benefício } \\
\text { significativo para salvar vidas para } \\
\text { qualquer tratamento. Ensaios clínicos } \\
\text { são necessários para orientar as } \\
\text { decisões de tratamento. }\end{array}$ & $\begin{array}{l}\text { Evidências de baixa qualidade } \\
\text { (com base em um único } \\
\text { estudo) indicam um benefício } \\
\text { do ECMO em casos graves de } \\
\text { MERS. Ensaios clínicos } \\
\text { colaborativos que avaliam } \\
\text { potenciais terapias são } \\
\text { urgentemente necessários para } \\
\text { orientar as decisões de } \\
\text { tratamento. }\end{array}$ \\
\hline 33 & $\begin{array}{l}\text { Characteristics and } \\
\text { outcomes of patients with } \\
\text { COVID-19 admitted to } \\
\text { hospital and intensive care } \\
\text { in the first phase of the } \\
\text { pandemic in Canada: a } \\
\text { national cohort study. } \\
\text { (MURTHY et al., 2021) }\end{array}$ & $\begin{array}{lr}\begin{array}{l}\text { Descrever } \\
\text { com }\end{array} & \text { pacientes } \\
\text { COVID-19 } \\
\text { internados na UTI, e } \\
\text { investigar preditores de } \\
\text { desfechor pra } \\
\text { caracterizar a SARA. }\end{array}$ & $\begin{array}{l}\text { Coorte } \\
\text { observacional } \\
\text { retrospectivo } \\
\text { multicêntrico }\end{array}$ & $\begin{array}{l}\text { Foram realizadas análises } \\
\text { descritivas de características, } \\
\text { intervenções e desfechos em } 811 \\
\text { pacientes de em média } 64 \text { anos. As } \\
\text { análises primárias examinaram a } \\
\text { mortalidade hospitalar, com } \\
\text { análises secundárias da duração da } \\
\text { UTI. }\end{array}$ & $\begin{array}{l}\text { Entre todos os pacientes, } 328 \text { receberam } \\
\text { atendimento em UTI, internados em } \\
\text { mediana de } 0 \text { dias. Pacientes gravemente } \\
\text { doentes receberam tratamento com VM, } \\
\text { terapia de reposição renal e ECMO; } \\
26,2 \% \text { morreram. Entre os que receberam } \\
\text { VM, } 31,2 \% \text { morreram. A mortalidade em } \\
\text { ECMO foi de } 69,2 \% \text {. }\end{array}$ & $\begin{array}{l}\text { Embora a ECMO possa ser } \\
\text { considerada para MERS grave, } \\
\text { neste caso a ECMO apresentou } \\
\text { uma taxa de mortalidade maior. }\end{array}$ \\
\hline
\end{tabular}




\begin{tabular}{|c|c|c|c|c|c|c|}
\hline 34 & $\begin{array}{l}\text { Timing of VV-ECMO } \\
\text { therapy implementation } \\
\text { influences prognosis of } \\
\text { COVID-19 patients. } \\
\text { (GIRAUD et al., 2021). }\end{array}$ & $\begin{array}{l}\text { Relatar a experiência, } \\
\text { princípios básicos, e } \\
\text { resultados no } \\
\text { estabelecimento } \mathrm{e} \\
\text { gerenciamento da } \\
\text { ECMO em pacientes } \\
\text { com COVID-19. }\end{array}$ & $\begin{array}{c}\text { Coorte } \\
\text { unicêntrico } \\
\text { retrospectivo }\end{array}$ & $\begin{array}{llr}\text { Foram analisados } 137 \text { pacientes } \\
\text { com SARA relacionada ao } \\
\text { COVID-19; internados na UTI dos } \\
\text { Hospitais Universitários de } \\
\text { Genebra. }\end{array}$ & $\begin{array}{l}\text { Dos } 137 \text { pacientes com SARA } \\
\text { internados na UTI, } 10 \text { pacientes } \\
\text { foram colocados em VV-ECMO. A } \\
\text { taxa de sobrevivência com ECMO } \\
\text { foi de } 40 \% \text {. Os sobreviventes tiveram } \\
\text { duração de VM menor antes da } \\
\text { ECMO. Todos os pacientes que } \\
\text { tinham mais de } 150 \mathrm{~h} \text { de VM antes } \\
\text { da aplicação do ECMO morreram. }\end{array}$ & $\begin{array}{l}\text { O VV-ECMO pode ser } \\
\text { utilizado com segurança em } \\
\text { pacientes com COVID-19 } \\
\text { selecionados com hipoxemia } \\
\text { refrataria. A } \\
\text { informação para os médicos é } \\
\text { que a terapia tardia de VV- } \\
\text { ECMO parece fútil. }\end{array}$ \\
\hline 35 & $\begin{array}{l}\text { Remdesivir for treatment of } \\
\text { COVID-19; an updated } \\
\text { systematic review and } \\
\text { meta-analysis. } \\
\text { (REZAGHOLIZADEH et } \\
\text { al., 2021) }\end{array}$ & $\begin{array}{l}\text { Avaliar a eficácia e a } \\
\text { segurança da } \\
\text { administração do } \\
\text { remdesivir em pacientes } \\
\text { com COVID-19. }\end{array}$ & $\begin{array}{c}\text { Revisão } \\
\text { sistemática e } \\
\text { meta análise }\end{array}$ & $\begin{array}{l}\text { Extração de dados publicados e } \\
\text { selecionados usando coleta de } \\
\text { dados da Colaboração Cochrane. O } \\
\text { cronograma de tratamento do } \\
\text { remdesivir nos estudos meta- } \\
\text { analisados foi na dose de } 200 \text { mgs } \\
\text { no primeiro dia, seguida de uma } \\
\text { dose de manutenção iv de } 100 \\
\text { mg/dia para os quatro a nove dias } \\
\text { subsequentes. }\end{array}$ & $\begin{array}{l}\text { Após uso do remdesivir houve suporte } \\
\text { (VM ou ECMO) durante os dias } 1 \text { a } 14 \text {. A } \\
\text { meta-análise dos grupos de remdesivir } \\
\text { demonstrou uma melhora significativa ao } \\
\text { longo do tempo em todas as categorias } \\
\text { avaliadas, exceto para a VM ou ECMO na } \\
\text { linha de base em comparação com o dia } \\
14 \text { e o suporte de oxigênio de baixo fluxo } \\
\text { na linha de base versus a análise do dia } \\
28 \text {. }\end{array}$ & $\begin{array}{l}\text { A administração do remdesivir } \\
\text { esteve associada à uma melhora } \\
\text { significativa na recuperação de } \\
28 \text { dias. A ECMO não mostrou } \\
\text { uma melhora eficaz. }\end{array}$ \\
\hline 36 & $\begin{array}{l}\text { Thrombotic complications } \\
\text { in } 2928 \text { patients with } \\
\text { COVID-19 treated in } \\
\text { intensive care: a systematic } \\
\text { review. } \\
\text { (JENNER } \text { et al., 2021) }\end{array}$ & $\begin{array}{l}\text { Avaliar a incidência de } \\
\text { complicações } \\
\text { trombóticas em } \\
\text { pacientes tratados na } \\
\text { UTI com COVID-19. }\end{array}$ & $\begin{array}{c}\text { Revisão } \\
\text { sistemática }\end{array}$ & $\begin{array}{l}\text { Foram selecionados estudos que } \\
\text { relataram complicações } \\
\text { trombóticas em pacientes com } \\
\text { COVID-19 internados na UTI. } \\
\text { Também foram incluídos estudos } \\
\text { que relataram pacientes que } \\
\text { receberam ECMO, dada a } \\
\text { consideração específica necessária } \\
\text { para a trombose nesses pacientes. }\end{array}$ & $\begin{array}{l}\text { Cinco estudos descreveram eventos } \\
\text { trombóticos em pacientes com ECMO. } \\
\text { No único estudo que realizou a triagem, } \\
\text { todos os } 13 \text { pacientes desenvolveram } \\
\text { tromboembolismo durante o ECMO. } \\
\text { Entre os } 51 \text { pacientes com ECMO onde } \\
\text { foi realizada a tomografia } \\
\text { computadorizada, 64\% tiveram relação } \\
\text { com a trombose. }\end{array}$ & $\begin{array}{l}\text { O uso da ECMO } \\
\text { relacionado com } \\
\text { tromboembolismo } \\
\text { pacientes. }\end{array}$ \\
\hline
\end{tabular}

Fonte: Autores (2022). 


\section{Discussão}

O presente estudo apresenta um compilado de informações sobre o uso da terapia ECMO em pacientes críticos com COVID-19, discutindo sua possível efetividade na evolução da doença. Os resultados desta revisão indicam primeiramente que, nos estudos analisados, houve maior perspectiva do benefício da terapêutica ao paciente, embora alguns sugiram que possa haver piora com o uso da terapia.

Dos 36 artigos elegíveis, 14 abordaram de forma direta o uso da ECMO em pacientes com COVID-19, enquanto 22 abordaram indiretamente o uso desta terapia.

Quando analisados os desenhos de estudo (método), foram incluídos dezoito artigos observacionais retrospectivos (4,9$12,15,18,19,21-23,26,27,29-31,33,34)$, quatro artigos de estudo prospectivo ${ }^{(3,13,20,24)}$, doze artigos de revisão sistemática ${ }^{(2,5-7,14,16,17,25,28,32,35,36)}$ e dois artigos ${ }^{(1,8)}$ de estudo randomizado.

Já em relação ao local de realização dos estudos, vinte e seis artigos (1,2,4-7,10,12-17,19-21,24-26,28-30,32,33,35,36) eram multicêntricos e dez artigos ${ }^{(3,8,9,11,18,22,23,27,31,34)}$ eram unicêntricos.

Analisando a eficácia da terapia em pacientes críticos com a COVID-19, vinte e cinco artigos (1-9,11,14,16-20,23-28,31,32,36) indicam benefícios do uso ECMO e um artigo ${ }^{(13)}$ considerou a ECMO como uma estratégia de resgaste razoável, totalizando 26 artigos que relataram benefício.

Esses dados de benefícios são baseados na eficácia da ECMO em manter o suprimento de oxigênio para os órgãos, evitando a falha de múltiplos órgãos, ${ }^{(2,17,19,25)}$ parada cardíaca, insuficiência respiratória e o agravamento da SARA ${ }^{(14)}$. A terapia também parece ser efetiva para prevenir os aspectos prejudiciais da VM, ajudando os pacientes a superarem a cascata inflamatória e economizando tempo para um tratamento clínico adicional ${ }^{(23)}$. Foi descrito também que a ECMO promove melhor recuperação pulmonar em pacientes em que as demais técnicas de ventilação de proteção não foram efetivas, como no caso da VM ${ }^{(28)}$. Além disso, em um artigo específico, foi mostrado que em dois de cinco pacientes que utilizaram a ECMO, houve a redução da Lactato desidrogenase, enzima que está associada a quadros de isquemia tecidual ${ }^{(27)}$.

No entanto, desses 26 artigos que indicam benefício, $3^{(3,9,36)}$ sugerem eficácia da terapia, porém, alto risco de tromboembolismo com o seu uso.

Foi verificada taxa significativamente maior de complicações trombóticas no circuito ECMO de pacientes COVID-19 (3,5,9,22,24,36) , com coágulos provenientes na maioria dos casos, da cabeça da bomba centrífuga, mas ocorrendo também nos conectores do circuito, nas cânulas e no oxigenador ${ }^{(3,9,36)}$. A formação desses coágulos, se não detectada, pode resultar em falha completa do sistema devido ao congestionamento do circuito ou superaquecimento do eixo da bomba ${ }^{(3)}$. Ressaltam também que devido essa tendência ao tromboembolismo, aplica-se por exemplo a heparina para a compensação, embora em alguns casos o uso destes anticoagulantes durante o tratamento ${ }^{(24)}$ acabe por gerar hemorragia durante o uso da ECMO, a qual pode ser observada em três artigos ${ }^{(9,11,29)}$.

Outros dois artigos ${ }^{(16,20)}$ relataram a ECMO como uma terapia que pode ser considerada para gestantes, pois evita a falha múltiplos órgãos, levando em consideração certos pontos como a idade gestacional, viabilidade fetal, bem-estar fetal e a saúde entre mãe e feto.

Ainda em relação à avaliação da efetividade do uso da ECMO em pacientes críticos com COVID-19, oito ${ }^{(10,12,15,21,30,33-}$ 35) indicaram tal inefetividade ou baixa eficiência.

Esta inefetividade, mesmo ainda não sendo esclarecida ${ }^{(15,21,34)}$, pode estar relacionada a vários fatores, incluindo a falha no oxigenador ${ }^{(11)}$. Alguns artigos relatam ainda a inefetividade da ECMO devido ao desenvolvimento de AVC hemorrágico ${ }^{(10,34)}$, pneumotórax, hipertensão pulmonar ${ }^{(12)}$, insuficiência renal, infecção secundária ${ }^{(19)}$, presença de linfocitopenia (baixa taxa 
de linfócitos), altas concentrações de Dímero- D e hipercapnia (alta taxa de CO2 no sangue), o que podem levar a um desmame malsucedido ${ }^{(30)}$. No entanto, no caso da associação com quadros trombóticos, como no AVC hemorrágico, e em outros eventos trombóticos evidenciados pelo aumento do Dímero-D, como já discutido anteriormente, há a possibilidade do uso de anticoagulantes para controlar esse efeito, embora o controle da medicação deva ser rigoroso para evitar quadros hemorrágicos.

Por fim, dois artigos ${ }^{(22,29)}$ consideraram inconclusiva a relação da eficácia da ECMO, sugerindo a necessidade de mais estudos.

Essa discrepância na avaliação da efetividade da ECMO pode ser devida a vários fatores.

Um dos principais é ao momento da doença em que foi utilizada, uma vez que cinco artigos ${ }^{(7,19,25,26,34)}$ apontaram a terapia como benéfica aos pacientes com COVID-19 e relataram que o uso precoce da terapia, ou seja, a utilização da ECMO de forma antecipada, está associada à melhora na sobrevida os pacientes. Estes estudos justificam que o uso precoce evita a progressão para falha de múltiplos órgãos do paciente em ECMO, enquanto o uso tardio da terapia chega a ser uma forma inútil para aumentar a sobrevida ${ }^{(7)}$.

Outra possibilidade para tal variação de resultados estaria relacionada com a idade e/ou comorbidades do paciente, havendo relatos de agravamento do risco do uso da ECMO em pacientes que possuem comorbidades como diabetes, hipertensão arterial, obesidade e idade avançada, mostrado em sete artigos $(7,13,21,23,25,26,28)$. No artigo 4 é apontado que pacientes com idade inferior a 71 anos tendem a ter maior sobrevida em relação àqueles com idade superior a 71 anos. $\mathrm{O}$ artigo 7 também relatou uma relação da mortalidade com pacientes que apresentavam erro de imunidade, mostrando a ECMO como não eficiente.

A associação à medicamentos também pode gerar resultados alterados, como mostrado nos artigos em que avaliaram o uso do Remdesivir ${ }^{(8,35)}$, mostrando que a ECMO foi eficaz associada a este medicamento antiviral, embora ainda não haja evidências concretas ${ }^{(8)}$. Neste mesmo artigo é mostrado uma melhora significativa do tempo de internação do grupo Redemsivir em comparação ao grupo placebo. No entanto, no artigo 35, o uso associado do Redemsivir e ECMO não apresentou melhora.

Já em relação à explicação para a associação da terapia ECMO com maior mortalidade em pacientes com COVID-19, também existem algumas variáveis a serem discutidas.

A inefetividade pode estar relacionada com a situação do paciente nas 24 horas que antecedem a canulação da ECMO, como quadros de linfocitopenia ${ }^{(28)}$, concentração elevada de dímero-D ${ }^{(3)}$ e hipercapnia, o que promove maior chance de um desmame malsucedido ${ }^{(11)}$. Estes valores, quando dentro dos padrões, são correlacionados às chances de um desmame bem sucedido da terapia.

Algo que também auxilia em um bom desmame, citado no artigo 30, é a realização da traqueostomia antes da descanulação da ECMO no paciente, e o uso de VM no momento pré-ECMO, o que também pode ser observado em 3 artigos ${ }^{(4,30,34)}$, como no caso do artigo 34, no qual pacientes que usaram VM por um menor tempo antes da ECMO tiveram mais sucesso no desmame em comparação àqueles que usaram a VM por um tempo maior.

\section{Considerações Finais}

Diante da revisão integrada, a maioria dos artigos relata a ECMO como uma técnica que pode trazer melhora aos pacientes críticos com COVID-19, principalmente com seu uso de forma precoce, em pacientes com idade inferior a 71 anos e com menos comorbidades. No entanto, ainda é mostrado que a terapia pode provocar aumento do risco de trombose, além de estudos que relatam inconclusão sobre a efetividade, sendo necessários mais estudos para investigar o uso dessa terapia nos pacientes com COVID-19. 
Research, Society and Development, v. 11, n. 3, e5911326388, 2022

(CC BY 4.0) | ISSN 2525-3409 | DOI: http://dx.doi.org/10.33448/rsd-v11i3.26388

Desse modo, embora a ECMO tenha se mostrado uma técnica que apresente benefícios nos pacientes com COVID-19, em especial se usada no início do tratamento, são necessários ainda mais estudos para avaliar a utilização desta técnica nestes pacientes, incluindo análise do momento de introdução e tipo de paciente a qual é indicada.

\section{Referências}

Bemtgen, X., Zotzmann, V., Benk, C., Rilinger, J., Steiner, K., Asmussen, A., ... Staudacher, D. L. (2021). Thrombotic circuit complications during venovenous extracorporeal membrane oxygenation in COVID-19. Journal of Thrombosis and Thrombolysis, 51(2) 301-307. doi: http://dx.doi.org/10.1007/s11239-020-02217-1

Beigel, J. H., Tomashek, K. M., Dodd, L. E., Mehta, A. K., Zingman, B. S., Kalil, A. C., ... Lane, H. C. (2020). Remdesivir for the Treatment of COVID-19 Final Report. England Journal Med, 383(19):1813-1826. doi: http://dx.doi.org/10.1056/NEJMoa2007764

Bleakley, C., Singh, S., Garfield, B., Morosin, M., Surkova, E., Mandalia, M. S., .. Price, S. (2021). Right ventricular dysfunction in critically ill COVID-19 ARDS. Journal Cardiologist, 327:251-258. doi: http://dx.doi.org/10.1016/j.ijcard.2020.11.043

Bergman, Z. R., Wothe, J. K., Alwan, F. S., Dunn, A., Lusczek, E. R., Lofrano, A. E., ... Brunsvold, M. E. (2021). The Use of Venovenous Extracorporeal Membrane Oxygenation in COVID-19 Infection: One Region's Comprehensive Experience. Ansaio Journal, 67(5):503-510. doi: http://dx.doi.org/10.1097/MAT.0000000000001403

Chaves, R., Filho, R. R., Timenetsky, K. T., Moreira, F. T., Vilanova, L. C.S., Bravim, B. A., ... Corrêa, T. D. (2019) Extracorporeal membrane oxygenation: a literature review. Hospital Israelita Albert Einsten, 31(3):410-424. Brasil. doi: http://dx.doi.org/10.5935/0103-507X.20190063

Coronavirus Resource Center. University and Medicine John Hopinks, EUA, 2021.Recuperado de: https://coronavirus.jhu.edu/

Davies, P., Evans, C., Kanthimathinathan, H. K., Lillie, J., Brierley, J., Waters, G., ... Ramnarayan, P. (2020) Intensive care admissions of children with paediatric inflammatory multisystem syndrome temporally associated with SARS-CoV-2 (PIMS-TS) in the UK: a multicentre observational study. Lancet Child Adolesc Health. Reino Unido, 4(9):669-677. doi: http://dx.doi.org/10.1016/S2352-4642(20)30215-7.

Donatelli, F., Miceli, A., Glauber, M., Cirri, S., Maiello, C., Coscioni, E., ... Napoli, C. (2021). Adult cardiovascular surgery and the coronavirus disease 2019 (COVID-19) pandemic: the Italian experience. Interact Cardiovasc Thorac Surg, 31(6):755-762. doi: http://dx.doi.org/10.1093/icvts/ivaa186.

Fang, J., Li, R., Chen, Y., Qin, J., Hu, M., Huang, C, L., ... Wei, X. (2021). Extracorporeal Membrane Oxygenation Therapy for Critically Ill Coronavirus Disease 2019 Patients in Wuhan, China: A Retrospective Multicenter Cohort Study. Curr Med Sci, 41(1):1-13. doi: http://dx.doi.org/10.1007/s11596-021-23118 .

Giraud, R., Legouis, D., Assouline, B., Charriere, A., Decosterd, D., Brunner M.-E., ... Bendjelid, K. (2021). Timing of VV-ECMO therapy implementation influences prognosis of COVID-19 patients. Physiol Rep, 9(3):e14715. doi: http://dx.doi.org/10.14814/phy2.14715

Guo, Z., Sun, L., Li, B., Tian, R., Zhang, X., Zhang Z., ... Li, X. (2020). Anticoagulation Management in Severe Coronavirus Disease 2019

Patients on Extracorporeal Membrane Oxygenation. Journal Cardiothorac Vasc Anesthesia, 35(2):389-397. doi: http://dx.doi.org/10.1053/j.jvca.2020.08.067

Hill, J. (1982) Classics in thoracic surgery John H. Gibbon, Jr. Part I. The Development of the First Successful Heart=Lung Machine. The Society of Thoracic Surgeons. Recuperado em: https://www.annalsthoracicsurgery.org/article/S0003-4975(10)62507-6/pdf

Hu, B.-S., Hu, M.-Z., Jiang, L-X., Yu, J., Chang, Y., Cao, Y., ... Dai, Z.-P. (2020). Extracorporeal membrane oxygenation (ECMO) in patients with COVID19: a rapid systematic review of case studies. European Review for Medical and Pharmacological Sciences, 24(22):11945-11952. China. doi: http://dx.doi.org/10.26355/eurrev_202011_23855

Jacobs, J., Stammers, A. H., Loius, J. S., Hayanga, J. W. A., Firstenberg, M. S., Mongero, L. B., ... Badhwar, V. J. (2021). Multi-institutional Analysis of 100 Consecutive Patients with COVID-19 and Severe Pulmonary Compromise Treated with Extracorporeal Membrane Oxygenation: Outcomes and Trends Over Time. Ansaio Journal, 67(5): 496-502. doi: http://dx.doi.org/ 10.1097/MAT.0000000000001434

Jenner, W. J., Kanji R., Mirsadraee, S., Gue, Y. X., Price, S., Prasad, S., ... Gorog, D. A. (2021). Thrombotic complications in 2928 patients with COVID-19 treated in intensive care: a systematic review. Journal Thromb Thrombolysis. 51(3):595-607. doi: http://dx.doi.org/10.1007/s11239-021-02394-7

Kache, S., Chisti, M. J., Gumbo, F., Mupere, E., Zhi, X., Nallasamy, K., ... Carcillo, J. (2020). COVID-19 PICU guidelines: for high- and limited-resource settings. Pediatric Research, 88(5):705-716. doi: http://dx.doi.org/ 10.1038/s41390-020-1053-9

Kaushik, S., Aydin, S., Derespina, K. R., Bansal, P. B., Kowals, S., Trachtman, R., ... Medar, S. S. (2020). Multisystem inflammatory syndrome in children associated with severe acute respiratory syndrome coronavirus 2 infection (MIS-C): A multi-institutional study from New York City. Journal Pediatric, 224: 24-29. Nova York. doi: http://dx.doi.org/10.1016/j.jpeds.2020.06.045

Köche, J. C. (2011). Fundamentos de metodologia científica. Editora Vozes. Retrieved from: https://www.academia.edu/download/38106165/192008010Fundamentos-de-Metodologia-Cien-Jose-Carlos-Koche-pdf_1.pdf.

Kowalewski, M., Fina, D., Stomka, A., Raffa, G. M., Martucci, G., Coco, V. L., ... Lorusso, R. (2020). COVID-19 and ECMO: the interplay between coagulation and inflammation-a narrative review. Critical Care. Holanda. https://www.ncbi.nlm.nih.gov/pmc/articles/PMC7209766/pdf/13054_2020_Article_2925.pdf

Lei, C., Lin, W., Hu F., Chen, F., Cai, W., Li, Y., ... Li, L. (2020). Factors associated with clinical outcomes in patients with Coronavirus Disease 2019 in Guangzhou, China. Journal Clin Virol, 133:104661. doi: http://dx.doi.org/10.1016/j.jcv.2020.104661 
Research, Society and Development, v. 11, n. 3, e5911326388, 2022

(CC BY 4.0) | ISSN 2525-3409 | DOI: http://dx.doi.org/10.33448/rsd-v11i3.26388

Liu, J., Dong, Y.-Q., Yin, J., He, G., Wu, X., Li, J., ... He, X. (2020). Critically ill patients with COVID-19 with ECMO and artificial liver plasma Exchange. Medicine Baltimore, 99(26):e21012. doi: http://dx.doi.org/10.1097/MD.0000000000021012

Martinelli, A, W., Ingle, T., Newman, J., Nadeem, I., Jackson, K., Lane, N. D., ... Marciniak, S. J. (2020). COVID-19 and pneumothorax: a multicentre retrospective case series. European Respiratory Journal, 56(5):2002697. Reino Unido. doi: http://dx.doi.org/10.1183/13993003.02697-2020

Meyts, I., Bucciol, G., Quinti, I., Neven, B., Fischer, A., Seoane, E., ... Tangye, S. (2020). Coronavirus disease 2019 in patients with inborn errors of immunity: An international study. Journal Allergy Clin Immunology, 147(2):520-531. doi: http://dx.doi.org/10.1016/j.jaci.2020.09.010

Murthy, S., Archambault, P. M., Atique, A., Carrier, F. M., Cheng, M. P., Codan, C., ... Wood, G. (2021). Characteristics and outcomes of patients with COVID19 admitted to hospital and intensive care in the first phase of the pandemic in Canada: a national cohort study. CMAJ Open, 9(1):E181-E188. doi: http://dx.doi.org/10.9778/cmajo.20200250

Mulcahy, C., Smith, M. G., Mamidi, I. S., Thakkar, P. G., Edwards, H., Tummala, N., .. Tracy, L. F. (2020). Oropharyngeal hemorrhage in patients with COVID-19: A multiinstitutional case series. Am Journal Otolaryngol, 41(6):102691. doi: http://dx.doi.org/10.1016/j.amjoto.2020.102691

Parzy, G., Daviet, F., Puech, B., Sylvestre, A., Guervilly, C., Porto, A., ... Forel, J.-M. (2020) Venous Thromboembolism Events Following Venovenous Extracorporeal Membrane Oxygenation for Severe Acute Respiratory Syndrome Coronavirus 2 Based on CT Scans. Critical Care Med, 48(10):e971-e975. França. doi: http://dx.doi.org/10.1097/CCM.0000000000004504

Radia, T., Williams, N., Agrawal, P., Harman, K., Weale, J., Cook, J., ... Gupta, A. (2020). Multi-system inflammatory syndrome in children \& adolescents (MIS-C): A systematic review of clinical features and presentation. Elsevier Public Health Collection, 38:51-57. doi: http://dx.doi.org/10.1016/j.prrv.2020.08.001

Ribeiro, T. (2020) Suporte Respiratório da COVID-19: qual o papel da ECMO. Jornal Brasileiro de Cirurgia Cardiovascular. Brasil. Recuperado em: https://blog.bjcvs.org/single-post/2020/04/03/suporte-respiratorio-na-COVID-19-qual-o-papel-da-ecmo/

Rieder, M., Wengenmayer, T., Staudacher, D., Duerschmied, D., \& Supady, A. (2020). Cytokine adsorption in patients with severe COVID-19 pneumonia requiring extracorporeal membrane oxygenation. Critical Care, 24(1):435. Alemanha. doi: http://dx.doi.org/10.1186/s13054-020-03130-y

Rezagholizadeh, A., Khiali, S., Sarbakhsh, P., \& Maleki, T. E. (2021). Remdesivir for treatment of COVID-19; an updated systematic review and meta-analysis. European Journal Pharmacol, 897:173926. doi: http://dx.doi.org/10.1016/j.ejphar.2021.173926

Roedl, K., Jarczak, D., Thasler, L., Bachmann, M., Schulte, F., Bein, B., ... Kluge, S. (2020). Mechanical ventilation and mortality among 223 critically ill patients with coronavirus disease 2019: A multicentric study in Germany. Aust Critical Care, 34(2):167-175. doi: http://dx.doi.org/10.1016/j.aucc.2020.10.009.

Sanders, J. M., Monogue, M. L., Jodlowski, T. Z., \& Cutrell, J. B., (2020). Pharmacologic Treatments and Supportive Care for Middle East Respiratory Syndrome. Emerg Infect Dis, 323(18):1824-1836. doi: http://dx.doi.org/10.1001/jama.2020.6019

Shaefi, S., Ma, X., Liang, M., Ding, M., Liu, W., Ma, H., Zhou, X., ... Ren, H. (2021). Extracorporeal membrane oxygenation in patients with severe respiratory failure from COVID-19. Intensive Care Med, 26:e925364. doi: http://dx.doi.org/10.12659/MSM.925364

Silva, ML, Alves, IOB, Ribeiro, CHMA \& Lo Prete, AC. Relação da coagulação intravascular disseminada pela determinação do dímero-D com a COVID-19: uma revisão integrativa. Research, Society and Development, 11(1), e21011225731, 2022. doi: http://dx.doi.org/10.33448/rsd-v11i2.25731

Supady, A., Taccone, F. S., Lepper, P. M., Ziegeler, S., \& Staudacher, D. L. (2021). Survival after extracorporeal membrane oxygenation in severe COVID-19 ARDS: results from an international multicenter registry. Critical Care, 25(1):90. doi: http://dx.doi.org/10.1186/s13054-021-03486-9

Sultan, I., Habertheuer, A., Usman, A. A., Kilic, A., Gnall, A., Friscia, M. E., ... Gutsche, J. (2020). The role of extracorporeal life support for patients with COVID-19: Preliminary results from a statewide experience. Wiley Public Health Emergency Collection, 35(7):1410-1413. doi: http://dx.doi.org/10.1111/jocs.14583

Savasi, V., Parisi, F., Patane, L., Ferrazzi, E., Frigerio, L., Pellegrino, A., ... Cetin, I. (2020). Clinical Findings and Disease Severity in Hospitalized Pregnant Women with Coronavirus Disease 2019 (COVID-19). Journal Logo, 136(2):252-258. doi: http://dx.doi.org/10.1097/AOG.0000000000003979

Suwalski, P., Rydzewski, A., Wojtowicz, R., Drobinski, D., Walecki, J., ... Wierzba, W. (2021). Severe respiratory failure in the course of coronavirus disease 2019 treated with extracorporeal membrane oxygenation. Kardiol Pol., 78(9):913-915. doi: http://dx.doi.org/10.33963/KP.15444

Tan, E., Song, J., Deane, A. M., \& Plummer, M. P. (2021). Global Impact of Coronavirus Disease 2019 Infection Requiring Admission to the ICU. Chest. 159(2):524-536. doi: http://dx.doi.org/10.1016/j.chest.2020.10.014

Umakanthan, S., Sahu, P., Ranade, A., Bukelo, M., Rao, J., Machado, L., ... KV, D. (2020). Origin, transmission, diagnosis and managemente of coronavírus disease 2019 (COVID-19). Postgrad Med J. Trindade e Tobago. Recuperado em: https://pmj.bmj.com/content/postgradmedj/96/1142/753.full.pdf.

Vogel, J. P., Tendal, B., Giles, M., Whitehead, C., Burton, W., Chakraborty, S., ... Homer, C. E. (2020). Clinical care of pregnant and postpartum women with COVID-19: Living recommendations from the National COVID-19 Clinical Evidence. Journal Obstet Gynaecol, 60(6):840-851. doi: http://dx.doi.org/10.1111/ajo.13270

Wang, M.-Y., Zhao, R., Gao, L.-J., Gao, X.-F., Wang, D.-P., ... Cao, J.-M. (2020). SARS-CoV-2: Structure, Biology, and structure-basaed therapeutics development. Frontiers in Cellular and Infection Microbiology. China. doi: http://dx.doi.org/10.3389/fcimb.2020.587269

Weiss, T. T., Cerda, F., Scott, J. B., Kaur, R., Sungurlu, S., Mirza, S. H., ... Li, J. (2021). Prone positioning for patients intubated for severe acute respiratory distress syndrome (ARDS) secondary to COVID-19: a retrospective observational cohort study. Br Journal Anaesth,126(1):48-55. doi: http://dx.doi.org/10.1016/j.bja.2020.09.042 
Research, Society and Development, v. 11, n. 3, e5911326388, 2022

(CC BY 4.0) | ISSN 2525-3409 | DOI: http://dx.doi.org/10.33448/rsd-v11i3.26388

Weber, A. G., Chau, A. S., Egeblad, M., Barnes, B. J., \& Janowitz, T. (2020). Nebulized in-line endotracheal dornase alfa and albuterol administered to mechanically ventilated COVID-19 patients: a case series. Mol Med. doi: http://dx.doi.org/10.1101/2020.05.13.20087734

Williams, G. W., Berg, N. K., Reskallah, A., Yuan, X., \& Eltzschinh, H. K. (2021). Acute Respiratory Distress Syndrome Contemporary Management and Novel Approaches during COVID-19. Anesthesiology, 134, 270-282. doi: http://dx.doi.org/10.1097/ALN.0000000000003571

Zhou, X., Cheng, Z., Shu, D., Lin, W., Ming, Z., Chen, W., ... Hu, Y. (2020). Characteristics of mortal COVID-19 cases compared to the survivors. Aging, 12(24):24579-24595. doi: http://dx.doi.org/10.18632/aging.202216 